\title{
Fixed and moored bodies in steep and breaking waves using SPH with the Froude-Krylov approximation
}

\author{
S. J. Lind ${ }^{1}$ - P. K. Stansby ${ }^{1}$ - B. D. Rogers ${ }^{1}$
}

Received: 2 December 2015 / Accepted: 28 April 2016 / Published online: 2 June 2016

(c) The Author(s) 2016. This article is published with open access at Springerlink.com

\begin{abstract}
Force prediction on fixed and moored bodies in steep, asymmetric and breaking waves remains a problem of great practical importance. For floating bodies snatch loads on mooring lines are of particular significance. In this paper we present an approximate approach where waves are modelled using incompressible smoothed particle hydrodynamics (SPH) which is well suited for breaking as well as non-breaking waves. For bodies of small size relative to wave length, the total force is assumed to be due to the Froude-Krylov force due to the undisturbed pressure field with additional added mass effects-in effect the Morison assumption. For a fixed vertical column in regular waves on a small slope, breaking wave force magnification is consistent with experiment and for focussed waves peak forces due to initial interaction are in good agreement with experiment; wave asymmetry is the dominant influence on overall force rather than local roller/jet breaker impact. For a taut moored hemispherical buoy in steep focussed waves the loads and motion without snatching are almost independent of added mass coefficient between zero and unity. Without breaking when snatching occurs the motion and loads measured experimentally are well predicted with zero added mass. This close agreement breaks down with wave breaking and the initial snatch load is overestimated by around $30 \%$. This approach
\end{abstract}

Research data supporting this publication is available from the 'Fixed and moored bodies using SPH with the Froude Krylov approximation' repository on figshare at https://dx.doi.org/10.6084/m9.figshare. 3370033.v2. This data is only available in a proprietary file format .lpk, which can be opened with Tecplot software.

$\triangle \quad$ S. J. Lind

steven.lind@manchester.ac.uk

1 Modelling and Simulation Centre, School of Mechanical, Aerospace and Civil Engineering, The University of Manchester, Manchester M13 9PL, UK is a fast alternative to fully 3-D simulations which are computationally demanding. Variation of, for example, mooring line properties and buoy position may be efficiently analysed using the same wave field and, as such, the approach has potential to be a useful design tool with further validation.

Keywords Incompressible smoothed particle hydrodynamics · Froude-Krylov force · Breaking wave forces · Focused waves $\cdot$ Snatch loads

\section{Introduction}

Wave forces on bodies have long been of interest in offshore and coastal engineering and are now of interest in marine and wind energy deployment. For example, the prediction of forces exerted on wind turbine columns is a vital design criterion. Breaking waves are likely, and, despite being the subject of sustained research for seven decades, there is still debate over the loads and forces exerted. For example, Miller et al. (1974) and Morison et al. (1953) reported that the post-breaking bore impact exerts the greatest force. However, Wienke and Oumeraci (2005) state loading is maximum at start of breaking where overturning begins and the incipient breaking jet remains horizontal. The issue is complicated by discussions around global and local forces, as the local force distribution on the cylinder is highly dependent on the wave profile and type of breaking wave impact: rise-times are short, pressure and force measurements are scattered, and air entrapment can play an important role at the impact location (Chan et al. 1995). While experimental studies are crucial in understanding loads due to wave breaking, engineering practice requires accurate modelling capabilities. The Morison force equation (Morison et al. 1950) is well-known and effective for small bodies if the wave is not breaking (Wiegel 
1982), and for input kinematics suitable non-linear potential flow solutions are available (Rienecker and Fenton 1981). An additional slam force can be added in the event of breaking, with a number of empirical parameters included such as the slam coefficient and curling factor [which depends on the orientation of the plunging jet (Goda et al. 1966; Watanabe and Horikawa 1974)]. Empirical approaches such as this are costeffective but require calibration and offer little insight into the underlying physics. Numerical simulation is the only alternative means to predict forces in flows that are highly deforming (and very non-linear), while simultaneously enabling insight into the underlying physics. However, for meaningful results one needs to calculate three-dimensional loads, and the spatial scale of the wave field often means the computational times are very long and the resources required considerable. Furthermore, modelling challenges exist in the accurate representation of the free surface and of breaking in the first instance. Classical numerical studies based on potential flow, like the boundary integral studies of Longuet-Higgins and Cokelet (1976) and Grilli et al. (2001), go some way towards modelling wave breaking but can only simulate a short time after overturning, before free-surface curvatures become prohibitively large or surfaces connect and the computation breaks down.

A full solution of the Navier-Stokes equations combined with the volume-of-fluid (VOF) method using CFD packages such as OpenFOAM (Christensen et al. 2005; Bredmose and Jacobsen 2010, 2011; Chen et al. 2014) or Ansys CFX (Hildebrandt and Schlurmann 2012) is a popular approach that can simulate wave breaking on cylinders and other bodies to good effect. However, VOF methods can have an overly diffusive interface, and the numerical implementation often requires a second air phase, which is not needed in the majority of the computational domain, if at all. In 3-D, this additional computational expense may be especially prohibitive. As an alternative, the numerical method smoothed particle hydrodynamics (SPH) has gained popularity in recent years. It is a Lagrangian particle method and, therefore, can model free-surface flows automatically while retaining a well-defined interface. It is a natural fit for wave breaking and wave impact studies and consequently has been applied to this area (Dalrymple and Rogers 2006; Shao 2006; Monaghan 1994; Lind et al. 2015), with improvement in water jet representation over conventional CFD demonstrated (Westphalen et al. 2014). However, in its original form SPH is weakly compressible, and consequently suffers from high-frequency spurious pressure oscillations due to the stiff equation of state (Lee et al. 2008). On the other hand, incompressible SPH (ISPH) has demonstrated accurate and effectively noise-free pressures for a range of internal and free-surface flows (both inviscid and highly viscous) (Lind et al. 2012): it is an ideal method to study loads due to wave breaking. However, compared to equivalent problems using finite volume methods, SPH is more computationally expensive due to the greater number of floating point operations per node point or "particle". Three-dimensional computations are particularly demanding and require optimised parallelisation on GPU (Crespo et al. 2015) or CPU (Guo et al. 2013) if simulation times are to remain practical.

In this paper, we present an alternative numerical approach within SPH using Froude-Krylov forcing to determine 3 -D loads on bodies due to 2-D plane incident wavesboth breaking and non-breaking. The effectiveness of this approach is demonstrated through comparisons with recent experimental work on breaking and non-breaking regular (Luck and Benoit 2004) and focused waves (Zang et al. 2010; Chen et al. 2014) on a vertical cylindrical column. The important case of an elastically moored floating body (buoy) is also considered, and comparisons are made with recent experimental measurements of focused wave interaction with a single-body bed-connected wave energy converter (WEC) (Hann et al. 2015). As with cylindrical columns, extremely large forces may be exerted on the device, and damage is a significant risk. Large and highly transient snatch loads in the elastic mooring line are a particular challenge, as would be experienced on the WEC in extreme sea-states. Mooring lines have recently been modelled and validated using SPH in still water and applied to moored vessel response in non-breaking waves (Aller 2015). Generally, floating bodies are challenging as the extreme response in the dynamically responding device may not coincide with the extreme properties of the wave [i.e. at the point of largest surface elevation (Taylor et al. 1997)]. Understandably, a means of accurately predicting the response and forces exerted on the device in extreme sea-states is necessary for informing future design and deployment to avoid failure in the mooring.

The Froude-Krylov-ISPH approach has a relatively low computational cost (requiring a two-dimensional simulation) and could be of interest in a number of practical engineering areas. Furthermore, multiple cylinder/buoy configurations can be considered rapidly in post-processing from a single incident wave simulation, as the flow is undisturbed with added mass effects accounted for through theoretical or empirically determined coefficients. The paper is structured as follows: Sect. 2 details the numerical method and the problem set-up, including details of wave generation and the Froude-Krylov implementation. Section 3 presents the results, including validations against linear wave theory, comparisons with the regular wave experiments of Luck and Benoit (2004), and comparisons with experimental data for focussed wave loads on cylinders (Zang et al. 2010; Chen et al. 2014). A selection of results on focused wave interaction (breaking and non-breaking) with a moored buoy (Hann et al. 2015) is presented in Sect. 4. Conclusions are made in Sect. 5 . 


\section{Numerical method}

\subsection{The problem setup and governing equations}

Consider a two-dimensional numerical wave basin of length $L$ and maximum water depth, $D$, as depicted in Fig. 1. A piston wave paddle is positioned at the left-hand side of the domain, centred at $x=0$, and is used to generate the required waves (regular or focused). A cylinder or buoy of diameter $d_{c}$ is centred at a distance $x_{c}$ from the origin. In the case of the buoy, $x_{c}$ is its initial horizontal location before displacement due to wave action. A schematic of the buoy model can be seen in Fig. 2. The buoy is comprised of a hemispherical base of radius $R_{b}$ with a cylindrical lid of height $H_{b}$, and is tautly moored to the bed with a mooring line of length $l_{m}$ and an anchoring that includes a load cell and joint (in experiment). The initial submerged depth of the buoy (draft) is denoted $D_{b}$. Two different mooring configurations are considered here with all physical parameter values provided in Sect. 4. For the cylinder cases, to enable breaking in the regular wave studies, as in the experiments of Luck and Benoit (2004), a $2.5 \%$ gradient ramp is inserted such that the initial local water depth at the cylinder is then $D_{\text {loc }}$.

The governing equations of a low-viscosity Newtonian fluid are to be solved: namely, the conservation of momentum,

$\frac{\mathrm{d} \mathbf{u}}{\mathrm{d} t}=-\frac{1}{\rho} \nabla p+v \nabla^{2} \mathbf{u}+\mathbf{g}$,

and the conservation of mass,

$\nabla \cdot \mathbf{u}=0$

The symbols $\mathbf{u}, p, \rho, v$, and $\mathbf{g}$ denote the fluid velocity, pressure, density, constant kinematic viscosity, and the acceleration due to gravity, respectively. The density and viscosity are assigned the physical constant values of $\rho=1000$ $\mathrm{kg} \mathrm{m}^{-3}$ and $v=1 \times 10^{-6} \mathrm{~m}^{2} \mathrm{~s}^{-1}$. The acceleration due to gravity, $\mathbf{g}$, is set to be $-9.81 \mathrm{~m} / \mathrm{s}^{2}$ vertically. On fixed solid boundaries the following boundary conditions are applied:

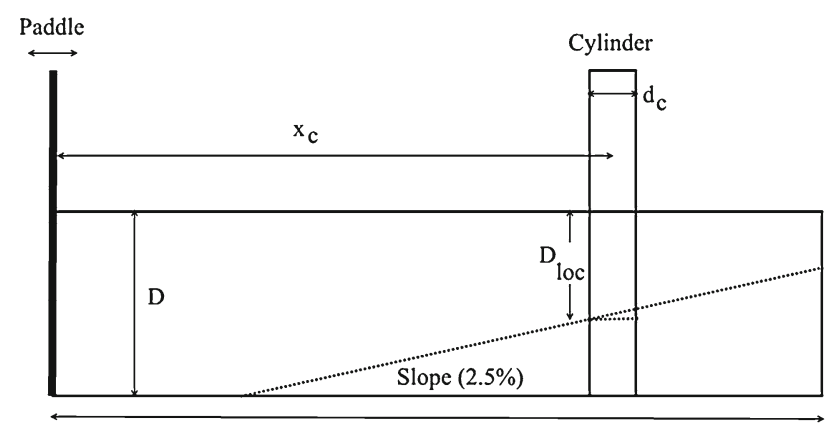

L

Fig. 1 Set-up of the numerical model used (not to scale). The ramp is only deployed in regular wave-cylinder studies

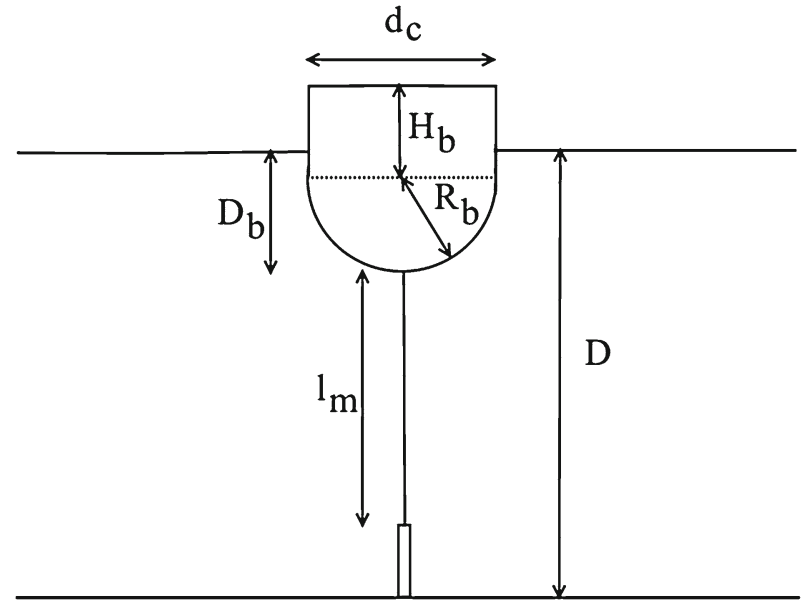

Fig. 2 Set-up of the buoy model used (not to scale). The mooring configuration varies, with dimensions and physical properties of the mooring provided in Sect. 4

$\mathbf{u} \cdot \mathbf{n}=0$ and $\frac{\partial p}{\partial n}=\rho \mathbf{g} \cdot \mathbf{n}$,

with atmospheric Dirichlet pressure boundary conditions $(p=0)$ imposed at the free surface. The mirror particle method (Morris et al. 1997) is used to impose boundary conditions on all solid boundaries except the ramp, which, when in use, is represented as a body of stationary dummy particles. Note that forces on the cylinder are calculated based on the undisturbed flow field [the Froude-Krylov force (Newman 1977)]: in effect the cylinder or buoy surface is "invisible" and does not require boundary conditions.

\subsection{Incompressible smoothed particle hydrodynamics}

\subsubsection{Particle interpolation and gradient calculation}

In $\mathrm{SPH}$, a variable $A$ at a point $\mathbf{r}$ is approximated by a convolution product of the variable $A$ with a smoothing kernel function $\omega_{h}\left(\left|\mathbf{r}-\mathbf{r}^{\prime}\right|\right)$, with a smoothing length $h$, and is written as

$A(\mathbf{r}) \approx \int_{\Omega} A\left(\mathbf{r}^{\prime}\right) \omega_{h}\left(\left|\mathbf{r}-\mathbf{r}^{\prime}\right|\right) d \mathbf{r}^{\prime}$,

where $\Omega$ is the supporting domain. When discretised over surrounding Lagrangian fluid particles the interpolation can be written as

$A\left(\mathbf{r}_{i}\right) \approx \sum_{j} V_{j} A\left(\mathbf{r}_{j}\right) \omega_{h}\left(r_{i j}\right)$,

where $V_{j}$ is the particle volume, $r_{i j}$ is the distance between particle $i$ and $j$. In this work, gradients are approximated using the zeroth-order consistent expression, 
$\nabla A\left(\mathbf{r}_{i}\right) \approx-\sum_{j}\left(A\left(\mathbf{r}_{i}\right)-A\left(\mathbf{r}_{j}\right)\right) V_{j} \nabla \omega_{h}\left(r_{i j}\right)$.

As in Xu et al. (2009) and Lind et al. (2012), all first derivative gradient (and divergence) approximations are used in combination with kernel gradient normalisation (Oger et al. 2007; Bonet and Lok 1998) to provide additional first-order consistency. Hereafter $\nabla \omega_{h}\left(r_{i j}\right)=\nabla \omega_{h}\left(\left|\mathbf{r}_{i}-\mathbf{r}_{j}\right|\right)$ will be simply written as $\nabla \omega_{i j}$ to denote an uncorrected kernel gradient, or $\nabla W_{i j}$, if a corrected gradient is used. In this paper a quintic spline kernel, continuous to the fifth derivative (Morris et al. 1997), is used for all cases, with a smoothing length of $h=1.3 d x$ (as widely used in the literature) given an initial particle spacing, $d x$.

\subsubsection{Enforcing incompressibility}

The incompressible governing Eqs. (1) and (2) are solved in exactly the same way as in $\mathrm{Xu}$ et al. (2009) and Lind et al. (2012). The volume, $V_{j}$, and density, $\rho_{j}$, of each SPH particle are taken to be constant. The projection method (Chorin 1968) is applied to integrate the incompressible governing Eq. (1) and enforces a divergence-free velocity field at each time step. The scheme used is overall first order in time and was first used within SPH by Cummins and Rudman (1999). First, particle positions, $\mathbf{r}_{i}^{n}$, are advected with velocity $\mathbf{u}_{i}^{n}$ to positions $\mathbf{r}_{i}^{*}$,

$\mathbf{r}_{i}^{*}=\mathbf{r}_{i}^{n}+\Delta t \mathbf{u}_{i}^{n}$

An intermediate velocity $\mathbf{u}_{i}^{*}$ is then calculated at the position, $\mathbf{r}_{i}^{*}$, based on the momentum equation without the pressure gradient term,

$\mathbf{u}_{i}^{*}=\mathbf{u}_{i}^{n}+\left(v \nabla^{2} \mathbf{u}_{i}^{n}+\mathbf{g}_{i}^{n}\right) \triangle t$.

The pressure at time $n+1$ can then be obtained from the pressure Poisson equation (PPE), written as

$\frac{1}{\rho} \nabla^{2} p_{i}^{n+1}=\frac{1}{\triangle t} \nabla \cdot \mathbf{u}_{i}^{*}$

An application of the Laplacian operator provided by Schwaiger (2008) and the SPH gradient approximation (6) results in the following discretised form of Eq. (9),

$$
\begin{aligned}
& \frac{\operatorname{tr}\left(\Gamma_{i}^{-1}\right)}{\rho}\left(\sum_{j} V_{j} \frac{p_{i j}^{n+1} \mathbf{r}_{i j} \cdot \nabla \omega_{i j}}{r_{i j}^{2}}-\nabla p_{i}^{n+1} \cdot \sum_{j} V_{j} \nabla \omega_{i j}\right) \\
& =-\frac{1}{\Delta t} \sum_{j} V_{j} \mathbf{u}_{i j}^{*} \cdot \nabla W_{i j},
\end{aligned}
$$

where $p_{i j}^{n+1}=p_{i}^{n+1}-p_{j}^{n+1}$ and the $(\alpha, \beta)$ th element of matrix $\Gamma_{i}$ is $\sum_{j} \mathbf{r}_{i j} \cdot \nabla \omega_{i j} \Delta x_{i j}^{\alpha} \Delta x_{i j}^{\beta} / r_{i j}^{2}$. Equation (10) forms a linear system for the pressure, $p_{i}^{n+1}$, that can be solved using an iterative solver (the stabilised bi-conjugate gradient method is used in this paper). The Dirichlet pressure boundary condition $(p=0)$ at the free surface is imposed within Eq. (10) on those particles satisfying the free-surface identity criterion of Lee et al. (2008); if the divergence of a particle's position vector is less than 1.6 then that particle resides on the free surface.

Following the solution of Eq. (10), the desired divergencefree velocity at time $n+1, \mathbf{u}_{i}^{n+1}$, then results from the projection of $\mathbf{u}_{i}^{*}$ :

$\mathbf{u}_{i}^{n+1}=\mathbf{u}_{i}^{*}-\frac{\triangle t}{\rho} \nabla p_{i}^{n+1}$

Finally, the particle positions are advanced in time,

$\mathbf{r}_{i}^{n+1}=\mathbf{r}_{i}^{n}+\Delta t\left(\frac{\mathbf{u}_{i}^{n+1}+\mathbf{u}_{i}^{n}}{2}\right)$

The above ISPH formulation uses the velocity divergence as the source term in the PPE. This formulation is known to be accurate over almost regular particle distributions, but can produce volume conservation errors and instability in particle methods in the long-term (Gui et al. 2014; Khayyer and Gotoh 2011). Khayyer and Gotoh (2011) have developed error mitigation terms for the source term which maintain both accuracy and stability, and improve volume conservation. These terms have since been applied to problems such as high-density multi-phase flows (Khayyer and Gotoh 2013) and violent sloshing (Gotoh et al. 2014), and to good effect. In this work, the velocity divergence source term provides accuracy in the flow field, with stability maintained through the use of Fickian particle shifting (Lind et al. 2012). Shifting is applied every time step and maintains an almost regular particle distribution in the fluid bulk, helping to preserve accuracy and conserve volume. When applied to ISPH, Fickian shifting has been shown to provide stable and accurate solutions for a wide range of Reynolds numbers without an apparent upper limit. The approach differs from other stabilising particle redistribution procedures (e.g. Xu et al. 2009) primarily in its applicability to free-surface as well as internal flows.

At free-surfaces, any shifting normal to the free surface is set to zero to prevent artificial diffusion of particles (and mass loss) through the free surface. This restriction means that, during the simulation, any new free-surface particles (which may migrate to the free surface in the presence of kernel truncation errors) become similarly restricted in normal movement. As all surface particles are prescribed a constant pressure, there is no hydrodynamic mechanism to regulate 


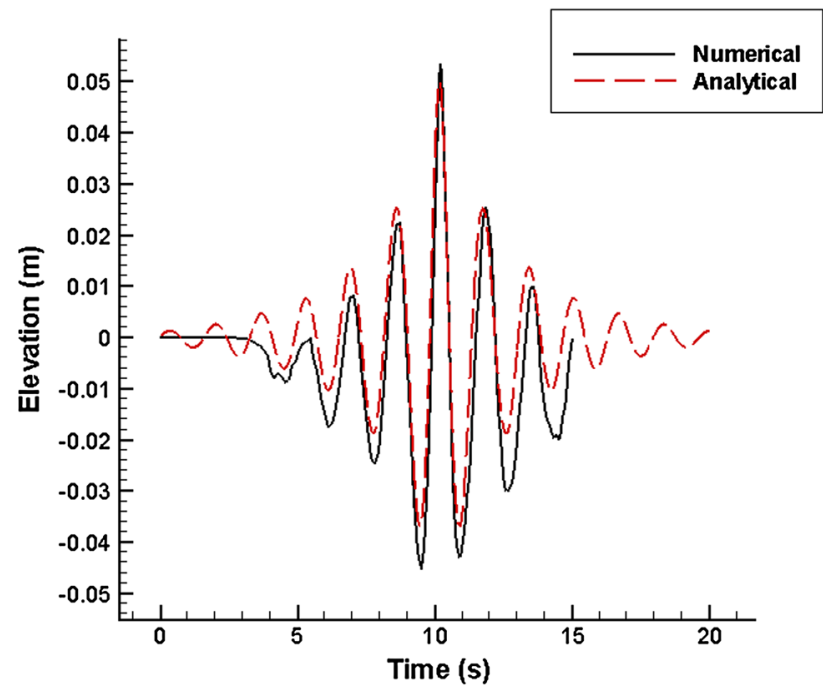

Fig. 3 Comparison between numerical (ISPH) and analytical (linear) results for the free-surface elevation of a JONSWAP wave group. Here $A=0.05 \mathrm{~m}, d x=0.01 \mathrm{~m}$

particle distributions around the free surface. Indeed, the only particle regularisation available is tangential shifting along the free surface. The effect of this restriction in the normal direction is a slight clustering of particles at the free surface resting above a gap of width less than $2 d x$. The effect is evident if one looks very closely at the free surface of Fig. 6, for example. It is important to emphasise that this phenomenon has negligible impact on the accuracy of the flow (as all particles approximating the free surface are assigned an analytical pressure Dirichlet boundary condition), and any small gap formed converges to zero as usual with particle refinement. For further details on the ISPH method employed herein, readers are referred to Lind et al. (2012) and Xu et al. (2009). For further detail regarding comparisons between different SPH projection methods and iterative solvers, readers are referred to $\mathrm{Xu}$ (2010).

\subsection{Wave spectra and generation}

As mentioned, two wave types are considered in this work: regular waves which may break on a slope, and irregular focused waves which may break depending on the superposition of a finite number of wave components at a focal point. A piston-type wavemaker is used to generate all waves, with its motion prescribed using linear wave theory. The linear analysis used for irregular focused waves is presented here, acknowledging that regular waves can be recovered by choosing a single wave component. The surface elevation, $\eta$, of an irregular wave is defined by the sum of harmonics

$\eta=\sum_{n} a_{n} \cos \left(k_{n} x-\omega_{n} t+\phi_{n}\right)$, where $a_{n}$ is the amplitude of the $n$th wave component and $k_{n}, \omega_{n}$, and $\phi_{n}$ are its associated wave number, frequency (angular) and phase, respectively. The "New Wave" group is employed (Tromans et al. 1991) where component amplitudes are calculated from a chosen wave spectrum according to the weighting,

$a_{n}=\frac{A_{N} S_{n} \Delta \omega_{n}}{\sum_{n} S_{n} \Delta \omega}$,

where $S_{n}$ is the power spectrum, $\Delta \omega$ is the frequency step, and $A_{N}$ is the maximum amplitude of the linearly superimposed wave components at a focal point, $x_{f}$. For the cylinder focused wave case a JONSWAP spectrum is used, with

$S \propto \frac{1}{\omega^{5}} \exp \left(-\frac{5}{4}\left(\omega_{p} / \omega\right)^{4}\right) \gamma^{r}$,

where

$\left.r=\exp \left(-\left(\omega-\omega_{p}\right)^{2} / 2 \sigma^{2} \omega_{p}^{2}\right)\right)$

with $\gamma=3.3$, and $\sigma=0.07$ if $\omega \leq \omega_{p}$ while $\sigma=0.09$ otherwise. Here $\omega_{p}$ is the frequency at the spectral peak. In contrast, the moored buoy study uses the Pierson-Moskowitz spectrum defined as above but for $\gamma=1$. Each spectrum was given a truncated frequency range $\omega_{\min }<\omega<\omega_{\max }$, with the values for $\omega_{\max }$ and $\omega_{\min }$ provided in the relevant results sections (guided by experiment). In order to focus the wave group at $x=x_{f}$ (with the wavemaker initially at $x=0$ ), the group celerity $c_{g}$ is calculated, based on the peak spectral frequency, and a focal time is specified, usually $2 x_{f} / c_{g}$. The phase angles, $\phi_{n}$, are then set to provide $\eta=A_{N}$ at the focal point. At the paddle wavemaker, the SPH mirror particle boundary conditions require the piston wavemaker velocity. The $n$th component of piston stroke amplitude, $s_{n}$, is given by

$\frac{s_{n}}{a_{n}}=\frac{\sin h\left(2 k_{n} D\right)+2 k_{n} D}{2\left(\cos h\left(2 k_{n} D\right)-1\right)}$,

where $D$ is still water depth (Dean and Dalrymple 1991). The piston velocity at $x=0$ thus given by

$u_{P}=\sum_{n} s_{n} \omega_{n} \cos \left(-\omega_{n} t+\phi_{n}\right)$.

For small-amplitude waves the above linear theory applied to the paddle is sufficient to generate the desired wave forms at a specified focal point. For larger amplitude waves $\left(A_{N} / D \gtrsim 0.1\right)$, non-linear wave interactions result in a focused wave around a shifted focal point that is markedly different in amplitude to linear predictions. Accordingly, for moderate to large amplitude focused waves, the amplitude 
is adjusted at the wavemaker through a process of trial and error (based on values provided by linear theory) until the wave elevations at a new focal point are in suitable agreement with experimental measurements for times at least up to wave focusing. The new focal point does not need to be matched to experiment as this is usually at the body which can be inserted at the new focal position (or any other location) for force calculation during post-processing. As in Lind et al. (2012), at the end of the wave tank a numerical damping zone is included to dissipate waves, with velocities reduced to zero according to the formula,

$\mathbf{u}=\mathbf{u}_{0}(1-\exp (-2(L-x)))$.

Studies have shown the above exponential damping over a length of $3 \mathrm{~m}$ (approximately the largest wavelength considered) is effective at dissipating energy and with negligible wave reflection effects at focusing.

\subsection{Froude-Krylov forcing}

In the experimental studies of interest, the KeuleganCarpenter numbers, $K C$, are moderate to small with $K C=$ $O(1)$. Reynolds numbers are also large $\left[R e=O\left(10^{5}\right)\right]$, meaning that inertial forces dominate the wave interaction (Sarpkaya and Isaacson 1981) as is often reported in experiments (e.g. Chen et al. 2014). Furthermore, the diameters of the cylinder and buoy are relatively small compared to wavelength (typically $d_{c} / \lambda \approx 0.2$ or less). Therefore, forces may be calculated using the Froude-Krylov approximation, with an appropriate added mass multiplier determined theoretically from potential flow (Newman 1977), or estimated empirically using experimental data. The Froude-Krylov (FK) force is given by the integration of the undisturbed pressure over the three-dimensional body surface, $C$ :

$\mathbf{F}_{F K}=-\int_{C} p \mathbf{n} d S$.

For the fixed cylinder case, classical potential flow analysis for a locally spatially uniform accelerating flow shows the additional force due to the flow disturbance around a cylinder is identical to that of the undisturbed flow field-equivalent to introducing an added mass equal to the fluid mass associated with the Froude-Krylov force (i.e. the added mass coefficient, $C_{a}=1$ ). Consequently, the total resultant horizontal force, $F_{x}$, for the cylinder cases can be taken as double its FK counterpart, viz.,

$F_{x}=-\left(1+C_{a}\right) \int_{C} p n_{x} d S=-2 \int_{C} p n_{x} d S$.

While estimation of the added mass based on potential flow is reasonable in non-breaking waves [and experimen- tally verifiable; see, for example, Keulegan and Carpenter (1958)], the use of a theoretical value of $C_{a}$ in the presence of breaking waves may be queried. However, it will be shown that the Froude-Krylov approximation with theoretically determined coefficients is quite accurate, even for an incident breaking wave. For example, the predicted peak horizontal loads due to the first breaking wave impact are within approximately $20 \%$ (or less) of experimental measurements for all focused wave cases. After all, the flow around the cylinder during the first breaking wave can remain very nearly irrotational (everywhere) up to the point of plunging jet break-up or crest spilling. After breaking, Nadaoka et al. (1989) used theory and experiment to determine the decomposed velocity field under a single post-breaking roller over intermediate depths, where it was shown that vorticity is primarily confined to the highly turbulent and aerated surf, with the subsurface flow remaining mostly irrotational. Numerical results are presented in Sect. 3 that support this observation. It is reasonable to postulate that the highly aerated and frothy surf imparts less momentum (and exerts less force) during cylinder interaction, compared to the (higher density) sub-surface irrotational flow. In other words, Froude-Krylov forcing with theoretical coefficients can still provide a reasonable approximation for total horizontal loads in early stage post-breaking waves.

It is expected that the FK approximation will worsen for waves in the later stages of breaking, and for any further breaking wave interactions after the first, as the sub-surface irrotational flow around the cylinder is increasingly disrupted and coherent 3-D structures (such as oblique descending eddies) can form in many cases (Farahani and Dalrymple 2014). The numerical results herein align with this expectation, and fully 3-D modelling will be necessary to accurately capture forces beyond the first couple of wave interactionsbut this is not the objective of this study. Indeed, a relatively quick and efficient numerical method that is able to predict even the first breaking wave load with reasonable accuracy is a useful capability in offshore engineering.

The above discussion also holds true for the buoy, but the buoy motion generates an additional added mass term with the unmoored translational force on the buoy approximated by,

$\mathbf{F}=\mathbf{F}_{F K}+\mathbf{C}_{\mathbf{a}} \rho V(\dot{\mathbf{u}}-\dot{\mathbf{v}})$,

where $\dot{\mathbf{v}}$ is the translational acceleration of the buoy at the centre of mass, while $\dot{\mathbf{u}}$ can be considered a volumetric average of the fluid acceleration over the wetted buoy volume, $V$. Here $\mathbf{C}_{\mathbf{a}}$ is an added mass matrix with diagonal components for heave and surge motions. For the buoy considered in this study, a WAMIT potential flow analysis (Lee 1995) yields added mass coefficients of 0.47 and 0.63 in heave and surge, respectively. Note that these values are determined for the 
buoy oscillating in a regular wave train at the peak frequency of the focused wave group used in experiment. However, it will be demonstrated that buoy response and mooring loads are largely insensitive to the choice of the added mass coefficient (in both heave, surge and pitch) as the effective force due to the relative motion of the buoy and surrounding fluid is small compared to the remaining hydrodynamic forces. Equivalently and quite conveniently, $\mathbf{C}_{\mathbf{a}}$ can be set to zero in the buoy case, giving good agreement with experiment.

The cylinder may be considered fixed, requiring a simple integration over a fixed three-dimensional cylindrical surface every time step. The buoy responds dynamically with the flow and has equations of motion that depend crucially on the mooring system in addition to hydrodynamic forces and gravity. The equations of motion for the buoy are thus (Ma and Yan 2009)

$M \dot{\mathbf{v}}=\mathbf{F}_{F K}+\mathbf{F}_{S}+m \mathbf{g}$,

and

$I \dot{\Omega}+\Omega \times I \Omega=\mathbf{N}$.

Here, $\mathbf{F}_{S}$ is the force due to tension in the mooring line, $m$ is the body mass ( $m=43.2 \mathrm{~kg}$ ), $\mathbf{N}$ is the moment arising due to hydrodynamics and the mooring line, and $\dot{\Omega}$ is the angular acceleration. The matrices $M$ and $I$ are the mass and moment of inertia matrices, respectively, and in general will include added mass and off-diagonal terms. As mentioned, an insensitivity to the added mass shall be presented in due course, while the planar nature of the flow and the symmetries in the buoy geometry enable off-diagonal terms in the inertia and mass matrices to be neglected. Indeed, $I$ effectively reduces to a single scalar (the pitch component), which, for the buoy in question, is calculated to be $I=1.61 \mathrm{kgm}^{2}$. The force $\mathbf{F}_{s}$ is that due to mooring tension and is governed by a damped elastic spring,

$\mathbf{F}_{s}=-k_{\mathrm{eq}} \mathbf{x}_{m}-c \dot{\mathbf{x}_{m}}$.

Here $k_{\text {eq }}$ is the equivalent spring constant determined from the system of lines defining the mooring, and $c=2 \zeta \sqrt{m k_{\mathrm{eq}}}$, where $\zeta$ is the damping ratio. Here $\mathbf{x}_{m}$ and $\dot{\mathbf{x}}_{m}$ are the mooring extension and rate of extension, respectively. The values of the constants $k_{\mathrm{eq}}$ and $\zeta$ will be discussed further in the results section for different mooring configurations, but it is worth noting that $k_{\text {eq }}$ is determined only from experimentally provided values. The moment $\mathbf{N}$ is that about the centre of mass due to hydrodynamical forces on the body and the mooring tension at the mooring connection point at the buoy's base, $\mathbf{r}_{b}$. Specifically,

$\mathbf{N}=\int_{C} p \mathbf{r} \times \mathbf{n} d S+\mathbf{r}_{b} \times \mathbf{F}_{s}$,
Table 1 Average relative error in pressure at front stagnation point at base of cylinder for various particle spacings

\begin{tabular}{ll}
\hline$d x(\mathrm{~m})$ & Av. relative error \\
\hline 0.005 & 0.019 \\
0.01 & 0.028 \\
0.02 & 0.059 \\
\hline
\end{tabular}

The presented error value is the mean of the relative errors calculated at times up to $10 \mathrm{~s}$

where $\boldsymbol{r}$ is a point on the buoy's surface relative to the centre of mass. The above equations are integrated in time during postprocessing using the already calculated flow data available at each time step. The velocity at any point on the buoy's surface, $\mathbf{v}_{\mathbf{r}}$ (and subsequently the buoy position/orientation), can determined using

$\mathbf{v}_{\mathbf{r}}=\mathbf{v}+\Omega \times \mathbf{r}$.

\section{Results: Part 1. Wave forces on a fixed cylinder}

\subsection{Validation against linear theory}

To validate the numerical method, Fig. 3 compares the freesurface elevation as predicted by ISPH, with the results from linear wave theory. The spectrum of the wave group is JONSWAP in shape with a peak frequency of $f_{p}=0.61 \mathrm{~Hz}$ over the range $(0.5-3) f_{p}$, and the peak amplitude at the focal point is $A_{N}=0.05 \mathrm{~m}$. The agreement in the peak amplitude and the location of the focal point is excellent. Note that the surface elevation is determined by taking the $y$ position of the free-surface particle closest to the focal point at each time step. Indeed, results show any discrepancy in the elevation remains small and within one particle spacing $d x=0.01 \mathrm{~m}$. Table 1 displays the corresponding relative error in pressure calculated at the front stagnation point at the base of a cylinder for several particle spacings. The error presented is an average of relative error values calculated at times up to $10 \mathrm{~s}$. Convergence is demonstrated with an order of convergence of approximately 1.0.

\subsection{Regular wave loading}

The first wave type to be considered and compared with experiment is that of a plane regular wave incident on a cylinder placed on a slope. The slope has a gradient of $2.5 \%$ and the cylinder (of diameter $0.2 \mathrm{~m}$ ) is positioned at $x_{c}=10 \mathrm{~m}$. Two water depths at the cylinder are considered, $D_{\mathrm{loc}}=0.4 \mathrm{~m}$ and $D_{\mathrm{loc}}=0.8 \mathrm{~m}$. Results are compared with the experimental measurements of Luck and Benoit (2004), and readers are referred to their paper for more information 


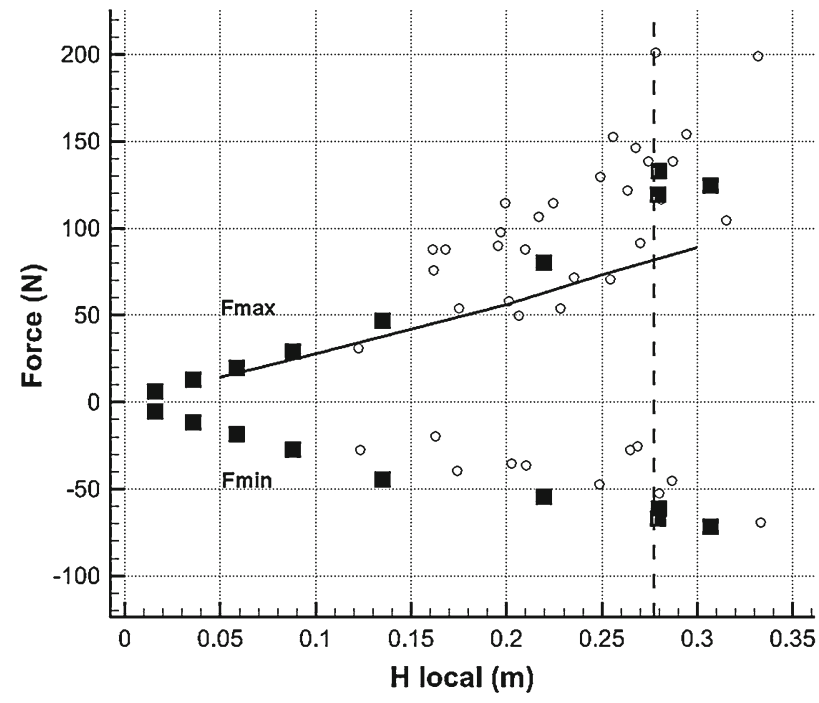

Fig. 4 Comparison with Luck and Benoit (2004) experimental results (circles) for loading on cylinder due to regular waves on a slope. The water depth at the cylinder is $0.4 \mathrm{~m}$. Black squares are ISPH, and the thick black line denotes predictions from SAWW. The vertical dashed line is the limiting wave height due to breaking at the cylinder according to Goda (1970)

on the experimental set-up. The first test case considers a regular wave of period $T=1.6 \mathrm{~s}$ with $D_{\mathrm{loc}}=0.4 \mathrm{~m}$ interacting with the cylinder for a range of (local) wave heights. Figure 4 presents the horizontal forces on the cylinder due to experiments of Luck and Benoit (2004) (circles), ISPH (black squares), and semi-analytical results (thick black line) from non-linear stream function theory for periodic water waves (Rienecker and Fenton 1981) used in combination with the Morison equation-as calculated in Stansby et al. (2013) using the SAWW software (Buss and Stansby 1982). The presented results from Luck and Benoit (2004) include several additional wave periods (up to $T=2.4 \mathrm{~s}$ ), but are suitably scattered so as to suggest no significant dependence of force on $T$. The ISPH results are the average of the first three wave impacts, to minimise the effect of spurious waves at paddle start up. For all wave heights, the ISPH results lie centrally within the experimental measurements recorded by Luck and Benoit (2004), and begin to deviate significantly from SAWW predictions for wave heights above $0.15 \mathrm{~m}$. Indeed, for heights above approximately $0.15 \mathrm{~m}$, the waves demonstrate significant asymmetry in their profile and may break at some point on the slope.

Figure 5 shows a typical incident wave crest on the cylinder with wave height $H=0.13 \mathrm{~m}$ (including pressure and horizontal velocity contours). The wave profile remains nearsymmetric and the wave unbroken. Figure 6 shows the wave profile (including pressure and horizontal velocity contours) for wave height $H=0.23 \mathrm{~m}$, where the wave is now asymmetric and near the point of overturning when striking the
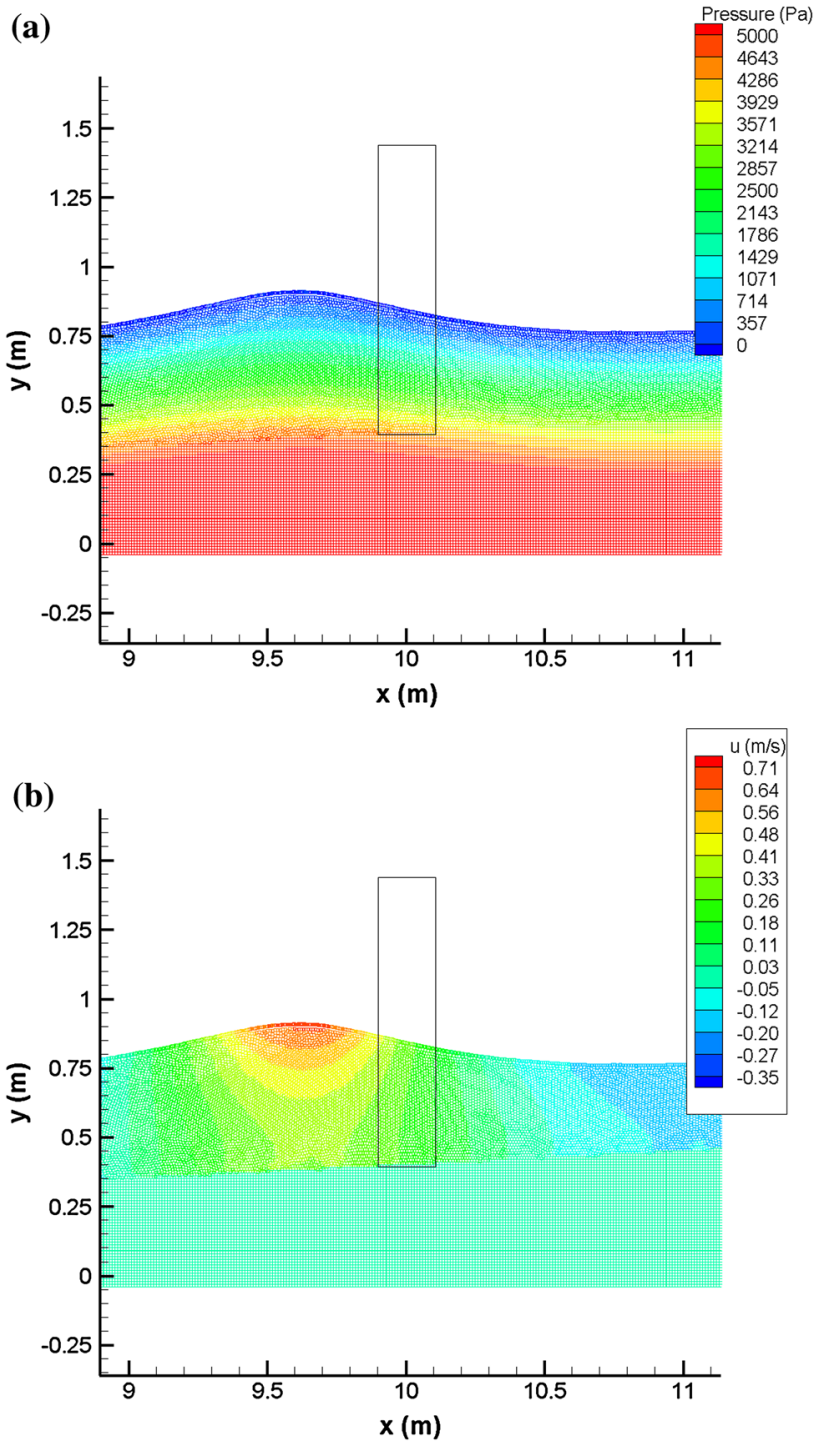

Fig. 5 Regular wave on a slope with pressure and velocity contours at cylinder for $H=0.13 \mathrm{~m}$ at time $t=7.8 \mathrm{~s}$. Water depth at cylinder $0.4 \mathrm{~m}$. a Pressure, $p$. b Horizontal velocity, $u$

cylinder. Indeed, the wave breaks fully just after the cylinder location. For a larger wave height $(H=0.3 \mathrm{~m}$, Fig. 7$)$, the wave has now broken before the cylinder in the form of a plunging breaker with spray. In fact, the wave height is so large for this case that an earlier smaller crest (formed during paddle start-up) formed a spilling breaker at a position slightly closer to the wave paddle. This explains the apparent fragmentation around the free-surface and the associated disturbance in the velocity contours for $x \leq 9.5 \mathrm{~m}$-it is a physical prediction of the surf layer formed from the first breaking event, as opposed to some non-physical numerical artefact.

The breaking events at $H=0.23 \mathrm{~m}$ and $H=0.3 \mathrm{~m}$ (Figs. 6 and 7, respectively) align quite closely with the empirical predictions of maximum wave height on a slope provided 

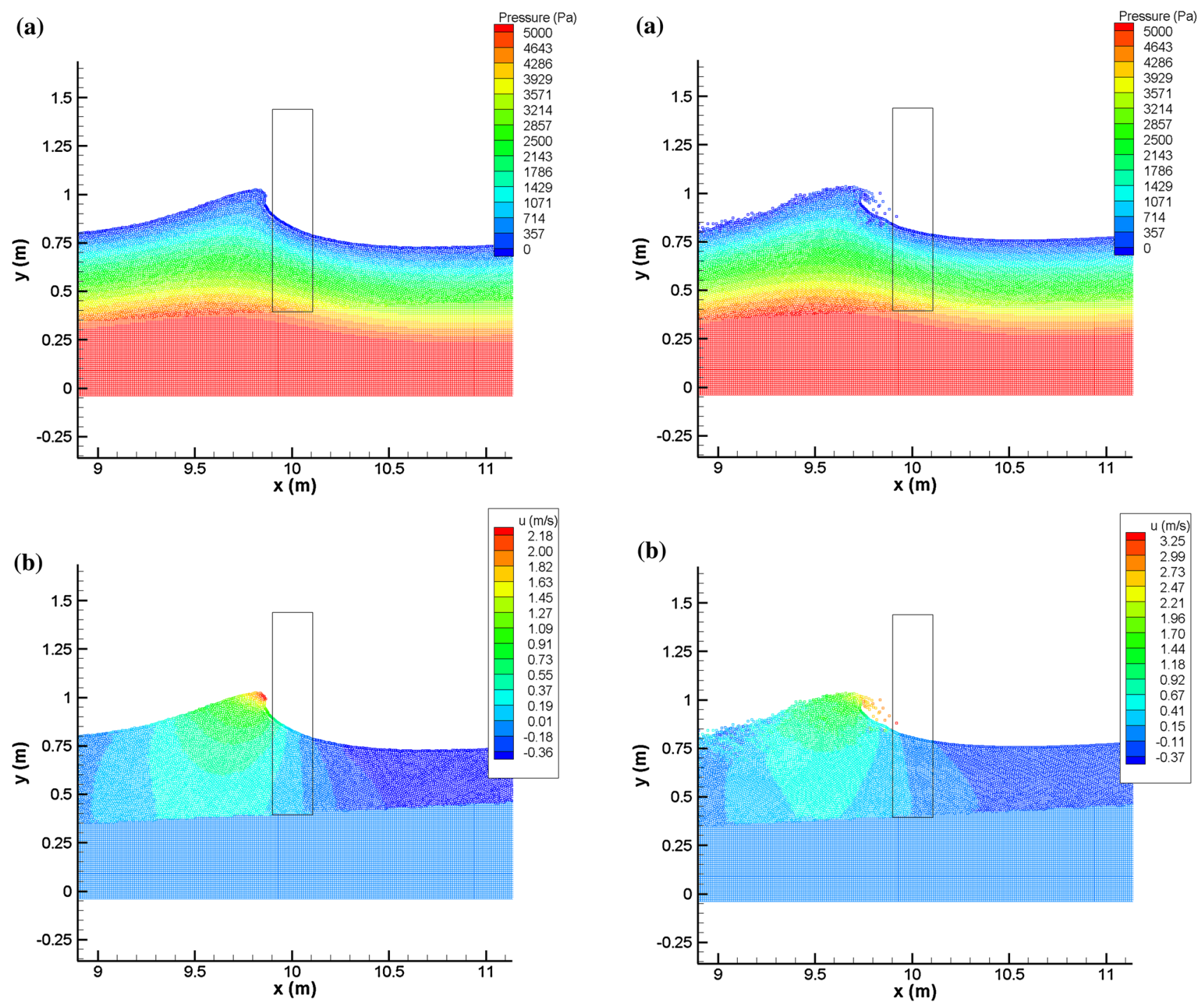

Fig. 6 Regular wave on a slope during overturning with pressure and velocity contours at cylinder for $H=0.23 \mathrm{~m}, t=9.2 \mathrm{~s}$. Water depth at cylinder $0.4 \mathrm{~m}$. a Pressure, $p$. b Horizontal velocity, $u$

by Goda (1970) (the vertical dashed line, Fig. 4). Note that the well-known Miche criterion for horizontal flat beds provides a maximum wave height within $3 \%$ of Goda (1970). Both asymmetric/breaking wave cases (Figs. 6,7) predict larger forces than SAWW, which provides a non-linear but symmetric profile for an equivalent wave height. The results are interesting for two reasons: Froude-Krylov forcing is sufficient to obtain good agreement with experiment, even in (early) breaking wave conditions. Secondly, results (both experimental and numerical) allude to amplification in the force due to asymmetry in the incident wave profile. In the following section, the force amplification due to breaking will be explored in more detail using results from a greater body of experimental data on focused waves. As seen in Fig. 8 , by increasing the water depth at the cylinder $\left(D_{\mathrm{loc}}=0.8\right.$

Fig. 7 Regular wave on a slope post-breaking with pressure and velocity contours at cylinder for $H=0.3 \mathrm{~m}, t=7.5 \mathrm{~s}$. Water depth at cylinder $0.4 \mathrm{~m}$. a Pressure, $p$. b Horizontal velocity, $u$

m) a reasonable agreement between all three force measurements (experimental, ISPH, SAWW) is now observed. Over approximately the same wave height range, the waves no longer break; all data now lie to the left of the Goda breaking criterion (Goda 1970) (vertical dashed line). The waves remain approximately symmetric about the wave crest and, accordingly, no significant amplification is observed in the applied load.

\subsection{Focused wave loading}

The second type of wave-cylinder interaction considered is that due to focused wave groups as in the experimental study detailed in Zang et al. (2010) and Chen et al. (2014) (undertaken at the DHI, Denmark). Surface elevations and 


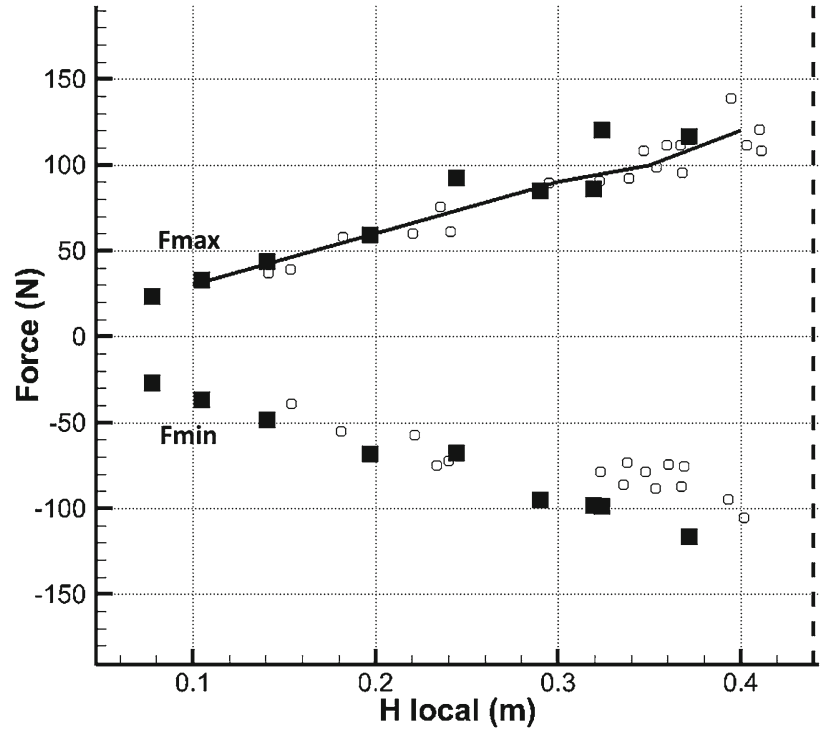

Fig. 8 As Fig. 4, but for local depth $D_{\text {loc }}=0.8 \mathrm{~m}$. The vertical dashed line is the limiting wave height due to breaking at the cylinder according to Goda (1970)

Table 2 Focused wave group cases considered in this study as in experiments of Zang et al. (2010) with the same case number notation

\begin{tabular}{lll}
\hline Case & Peak frequency $(\mathrm{Hz})$ & Wave height $(\mathrm{m})$ \\
\hline F11 & 0.82 & 0.07 \\
F3 & 0.61 & 0.12 \\
F16 & 0.61 & 0.23 \\
F14 & 0.82 & 0.14 \\
F13 & 1.22 & 0.1 \\
F15 & 0.82 & 0.22 \\
\hline
\end{tabular}

The wave height is that measured at the theoretical focal point at the cylinder front. The frequency is that of the peak in the JONSWAP spectrum

horizontal forces are available at the cylinder for six different wave groups of various frequencies and wave heights. Table 2 summarises the wave groups studied and the parameters used for each case. For consistency with obtained experimental results, we adopt an identical naming convention for each focused wave case (Fnumber). There is no slope in this case (see Fig. 1), as breaking waves may be formed by the focusing of the wave group into an unstable wave form at the front of the cylinder. The numerical wave tank is taken to be of length $L=12 \mathrm{~m}$ and still water depth $D=0.505 \mathrm{~m}$ (as in the experiment). The cylinder (of diameter $0.25 \mathrm{~m}$ ) is centred at $x_{c}=7.52 \mathrm{~m}$.

\subsubsection{Case F11: $H=0.07 \mathrm{~m}$}

First, consider a low-amplitude case (F11), where breaking does not occur (see Fig. 9). Figure 10 compares the ISPH and experimental predictions for the elevation at the front of the cylinder. The agreement is good (within one particle

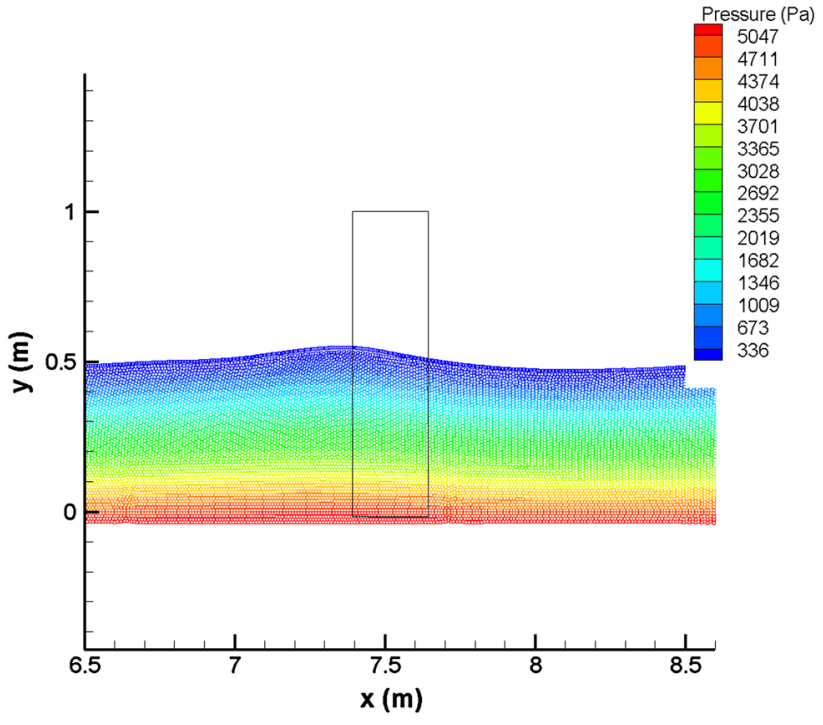

Fig. 9 Wave profile and pressure contours near the focal point for the F11 case (at time $t=13.26 \mathrm{~s}$ )

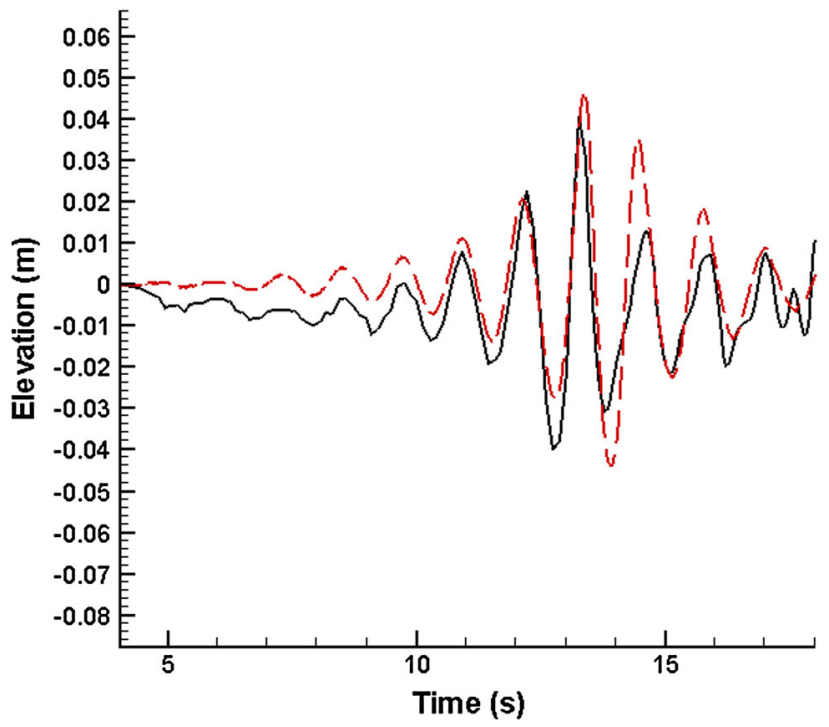

Fig. 10 Comparison between experimental and numerical results for the free-surface elevation at the front of the cylinder for focused wave case F11. The dashed lines denote experimental measurements, the black line is ISPH

spacing) up to the point of the peak (focused) wave crestcylinder interaction. After this point the agreement begins to worsen, but is still reasonable. Similarly, the small discrepancy visible in the mean water level remains within one particle spacing accuracy. The horizontal force exerted on the cylinder (Fig. 11) demonstrates a similar behaviour to the elevation-the agreement with experiment is very good up to and including the peak wave crest interaction, with agreement decreasing slightly thereafter. The increased discrepancy after the peak wave interaction (in both force and elevation) is understandable. There will be interaction effects 


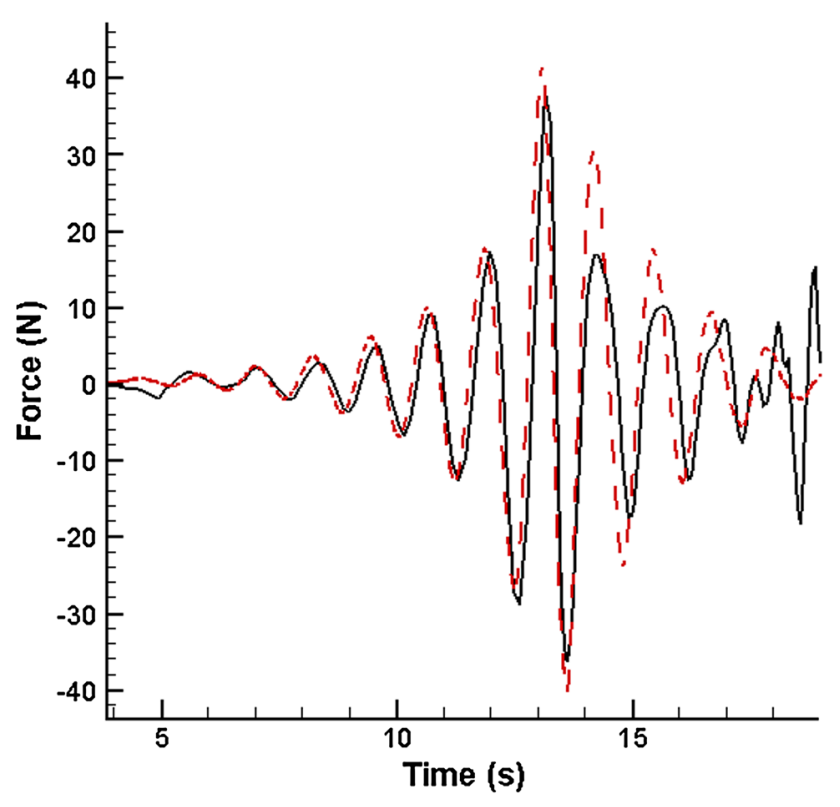

Fig. 11 Comparison between experimental and numerical results for the total horizontal force on the cylinder for focused wave case F11. The dashed lines denote experimental measurements, the black line is ISPH

(especially around the free surface) that are omitted entirely from the Froude-Krylov approximation, and not modelled through the inclusion of a theoretical added mass. The linear wave paddle and damping zone may also be a source of discrepancy at longer times. Importantly, however, predictions up to and including the peak wave interaction, where the largest and most relevant loads are exerted, are very good.

\subsubsection{Case F3: $H=0.12 \mathrm{~m}$}

The case F3 is another low to intermediate amplitude case with good agreement in both the focused wave elevations (Fig. 12) and the horizontal forces (Fig. 13). As can be seen from Fig. 14, the wave is of low amplitude and non-breaking. Note that a small phase difference compared with experiment is apparent in Fig. 12 (free-surface elevation) after $t \approx 10 \mathrm{~s}$ that is not observed in Fig. 13 (force). The reason for this is likely due to the method for plotting the elevation, where, as mentioned already, the $y$ coordinate of the free-surface particle closest to a fixed $x$ location (i.e. the cylinder front) determines the elevation at that fixed point. This procedure is sensitive to single particle positions and has a plotting error on the order of the particle spacing which can manifest as error in elevation and phase (for example, a crest may be measured to be at the cylinder front before actually arriving). This effect can then be compounded by actual numerical error in the position of the free surface. The force, on the other hand, is a far less sensitive measure as integration of pressure over the whole cylinder surface (and a comparatively

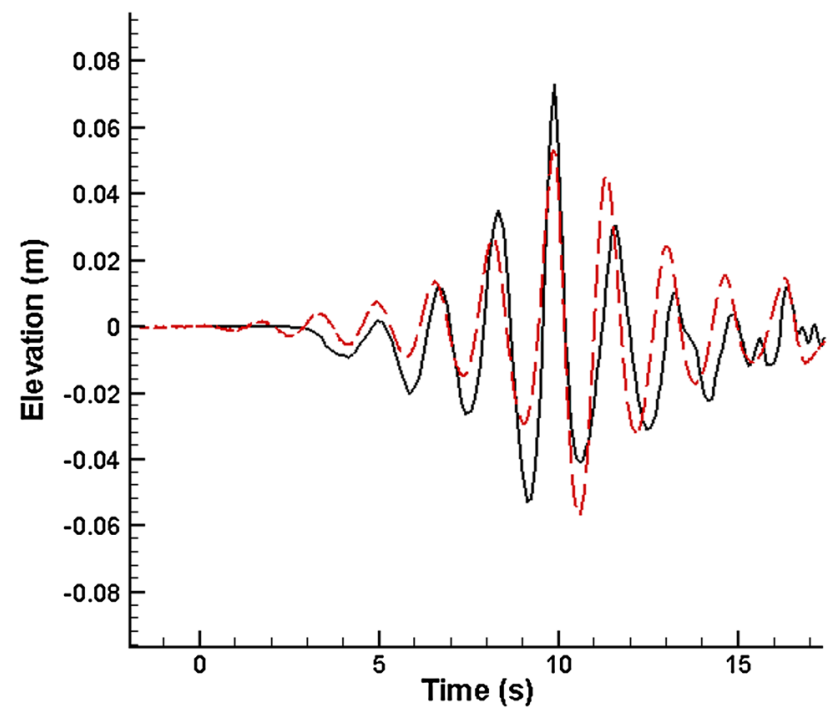

Fig. 12 Comparison between experimental and numerical results for the free-surface elevation at the front of the cylinder for focused wave case F3. The dashed lines denote experimental measurements, the black line is ISPH

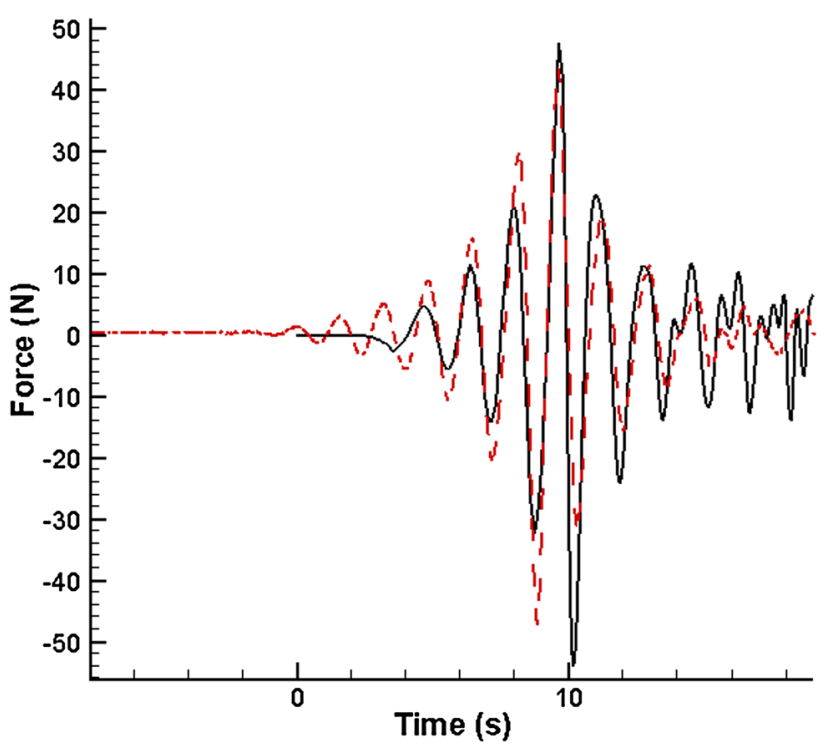

Fig. 13 Comparison between experimental and numerical results for the total horizontal force on the cylinder for focused wave case F3. The dashed lines denote experimental measurements, the black line is ISPH

large number of particles) dominates any local error due to particle position. Nevertheless, the results for both cases F11 and F3 are sufficiently accurate so as to suggest a validated numerical method. It also seems a sufficient spatial resolution $(d x=0.01 \mathrm{~m})$ is being used to predict accurate loads up to the peak wave crest interaction. A convergence study is undertaken below, see Fig. 30, further supporting this value for $d x$. Importantly, the use of a moderate resolution means a key feature of this numerical approach (relatively rapid 3-D load calculations) is maintained. 


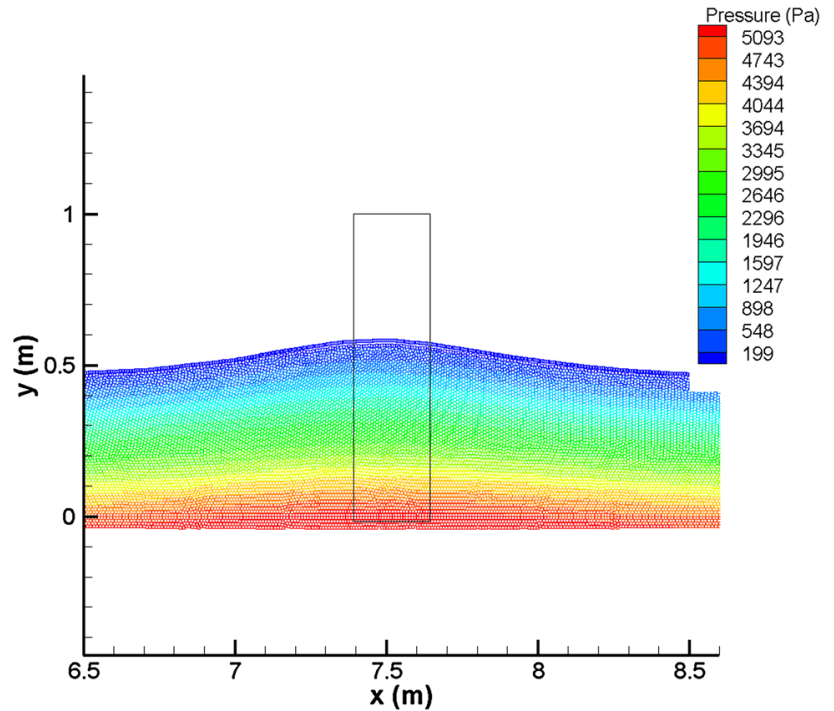

Fig. 14 Wave profile and pressure contours near the focal point for the F3 case $(t=9.88 \mathrm{~s})$

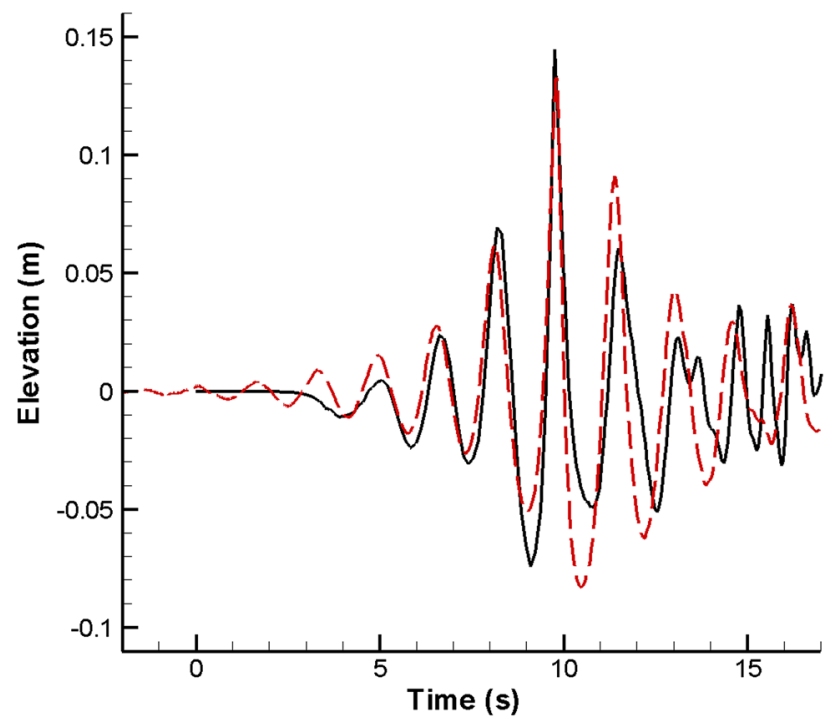

Fig. 15 Comparison between experimental and numerical results for the free-surface elevation at the front of the cylinder for focused wave case F16. The dashed lines denote experimental measurements, the black line is ISPH

\subsubsection{Case F16: $H=0.23 \mathrm{~m}$}

The next case (F16) considers a larger wave amplitude with $H=0.23 \mathrm{~m}$. As the amplitude is quite large, linear theory is fairly inaccurate in its predictions and the peak amplitude required at input is adjusted accordingly to reproduce the observed elevation at the cylinder as close as possible. Figure 15 shows the elevation at the cylinder front, using a calibrated input at the wavemaker. Expectedly elevations are in good agreement up to and including the peak wave interaction, and importantly the horizontal forces are also

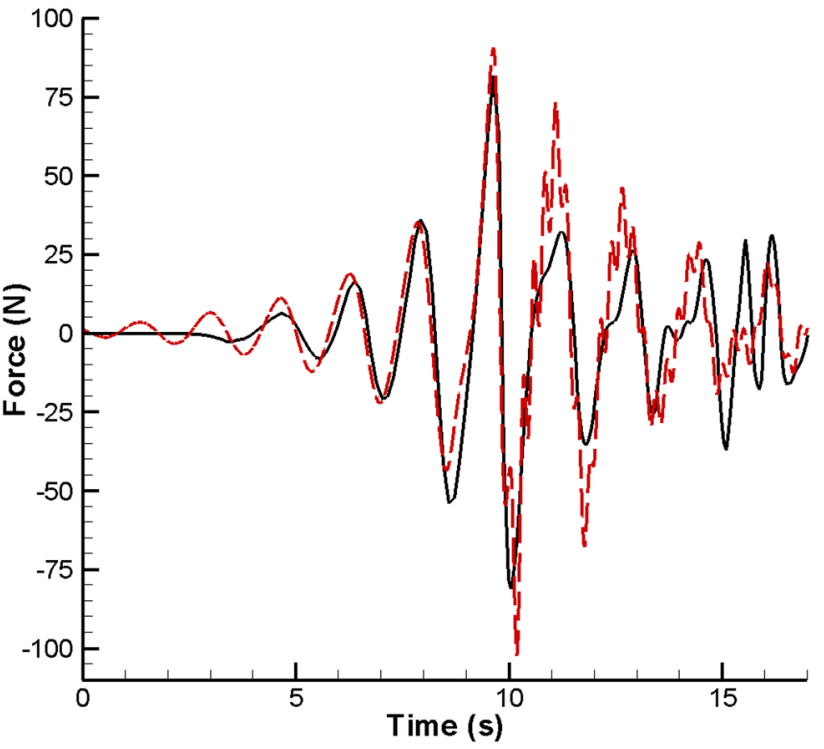

Fig. 16 Comparison between experimental and numerical results for the total horizontal force on the cylinder for focused wave case F16. The dashed lines denote experimental measurements, the black line is ISPH

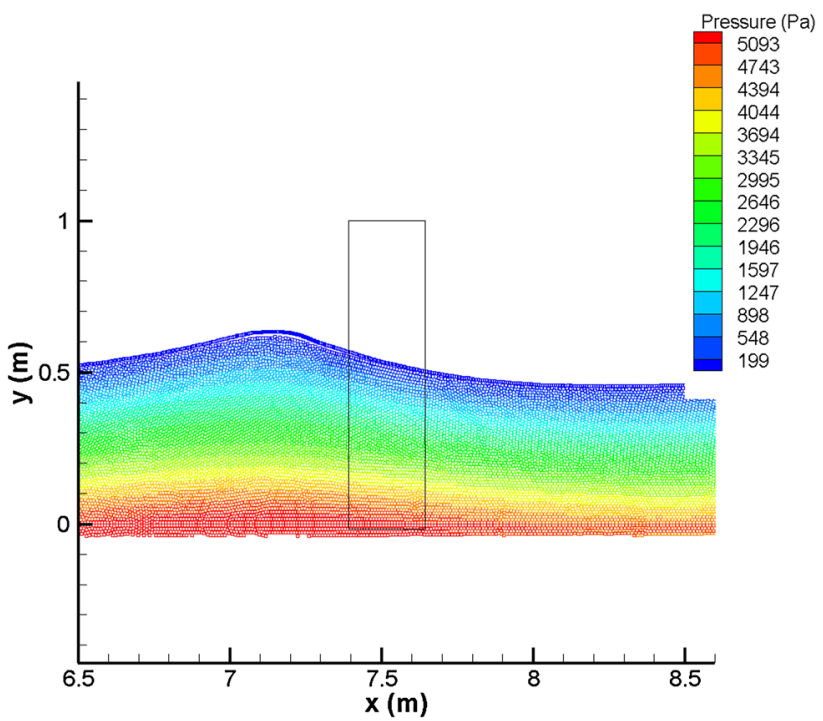

Fig. 17 Wave profile and pressure contours near the focal point for the F16 case $(t=9.62 \mathrm{~s})$

accurately predicted up to this point (Fig. 16). There are some high-frequency low-amplitude oscillations in the force after the peak in the experimental data, no doubt resulting from non-linear interaction effects not considered here. This is a large amplitude wave, but non-breaking, as can be seen in Fig. 17.

\subsubsection{Case F14: $H=0.14 \mathrm{~m}$}

The case F14 is of intermediate wave height, but with a peak frequency larger than the case F16. This is a breaking 


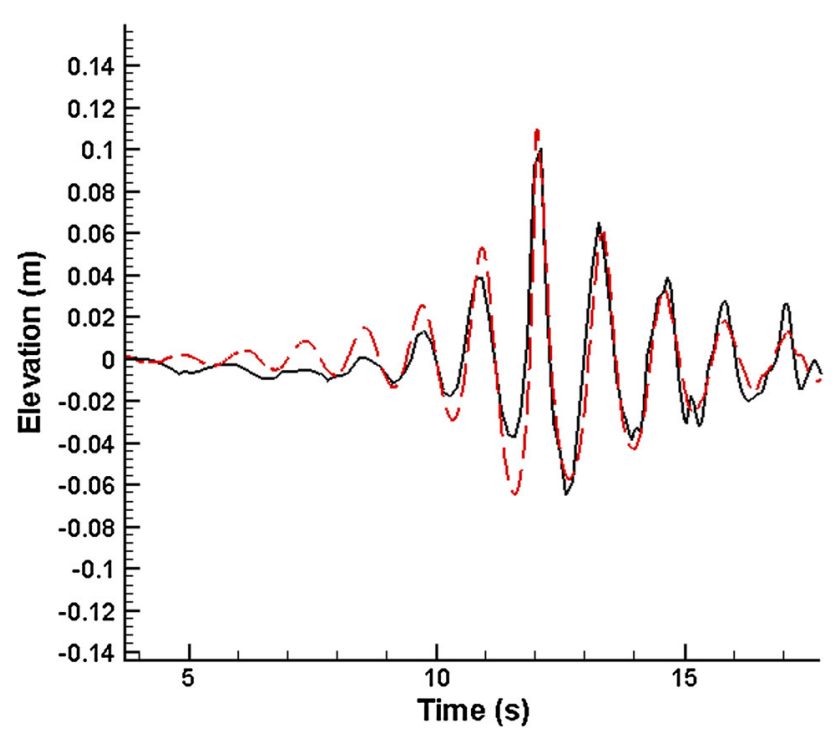

Fig. 18 Comparison between experimental and numerical results for the free-surface elevation at the front of the cylinder for focused wave case F14. The dashed lines denote experimental measurements, the black line is ISPH

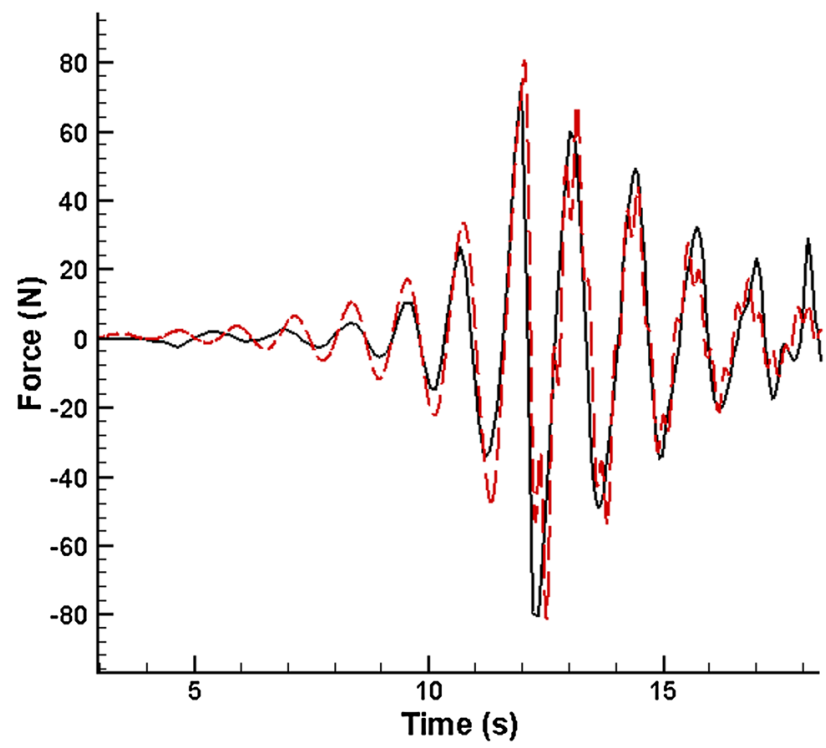

Fig. 19 Comparison between experimental and numerical results for the total horizontal force on the cylinder for focused wave case F14. The dashed lines denote experimental measurements, the black line is ISPH

wave case with a plunging breaker forming approximately $0.5 \mathrm{~m}$ before the front of the cylinder resulting in a postbreaking roller hitting the cylinder front. Figures 20 and 21 show the breaking process at select times with pressure and horizontal velocity contours, respectively. The free-surface elevation (Fig. 18) and force calculations (Fig. 19) are in good agreement with experiment, with local maxima and
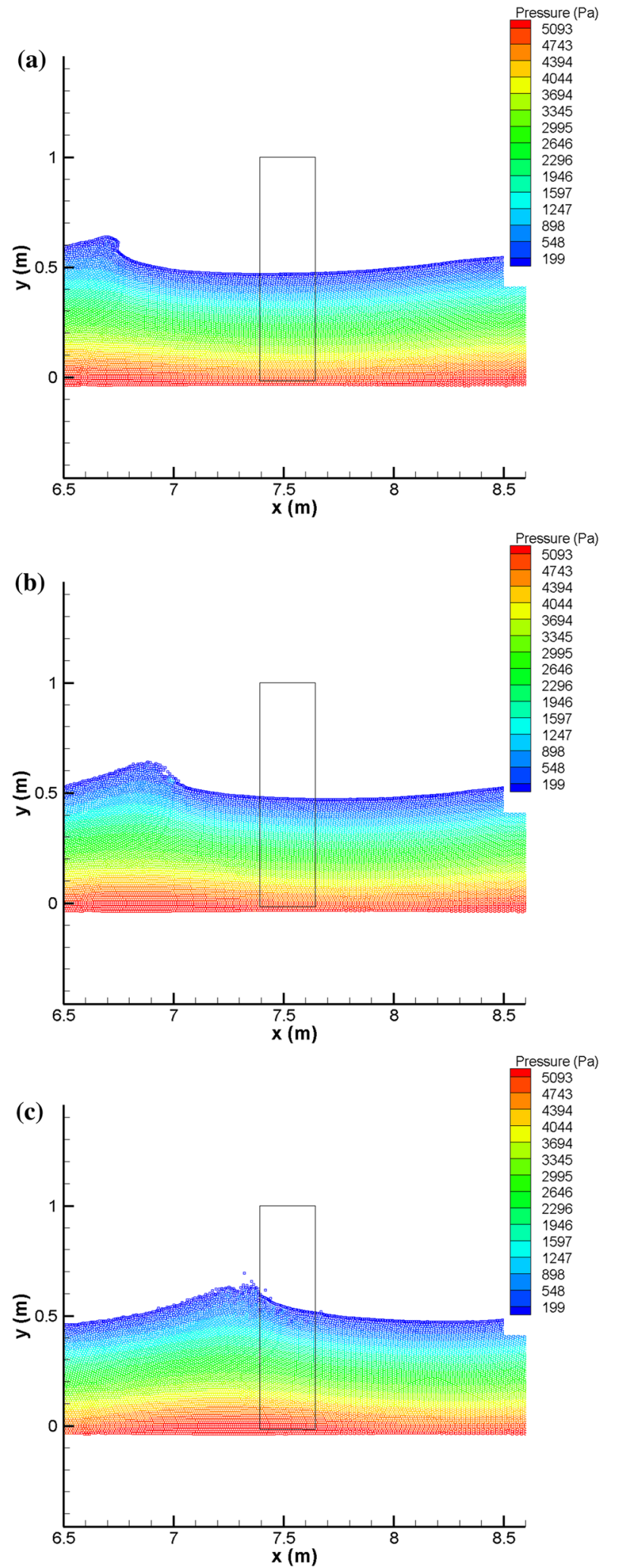

Fig. 20 Wave profile and pressure contours at select times near the focal point at the cylinder (case F14). a $t=11.70 \mathrm{~s}, \mathbf{b} t=11.83 \mathrm{~s}, \mathbf{c} t=$ $11.96 \mathrm{~s}$ 

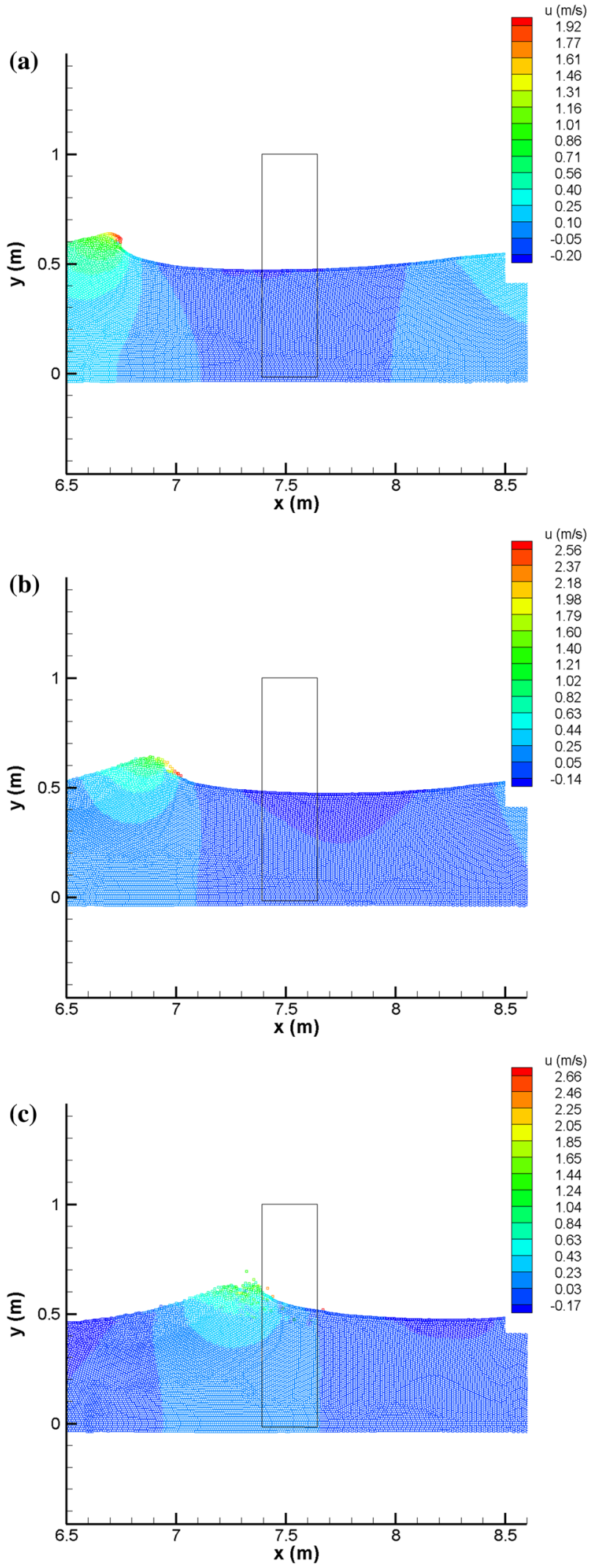

Fig. 21 As Fig. 20, but with horizontal velocity contours. a $t=11.70 \mathrm{~s}$, b $t=11.83 \mathrm{~s}, \mathbf{c} t=11.96 \mathrm{~s}$ minima captured before and after the roller-cylinder interaction. While the local wave height is similar to the case F3 (non-breaking), the recorded maximum force (both experimental and numerical) is approximately twice as large in this breaking wave case.

To support the justification in Sect. 2.4 on the use of a theoretical (potential flow) added mass coefficient for breaking wave-cylinder interaction, Fig. 22 plots contours in the vorticity magnitude over the same time intervals as Fig. 20. In line with the experimental observations of Nadaoka et al. (1989), as the wave breaks and the roller passes through the cylinder, the vorticity remains confined to a region less than one wave height from the free surface. As mentioned in Sect. 2.4, shortly after breaking this surf is highly aerated (in reality) and of relatively low pressure (as predicted here), and is likely to impart less momentum to the cylinder compared with the remaining sub-surface irrotational flow. In other words, it is reasonable to apply the Froude-Krylov force with theoretical coefficients to approximate global horizontal loads. Indeed, from wave theory it is known that the largest variation in non-hydrostatic pressure occurs at the bed, so while the added mass may deviate from theory in the surf, the FK force (Eq. 21) remains dominated by larger sub-surface pressure variation where use of a theoretical coefficient holds better. Note that the imposition of slip conditions on the bed precludes generation of vorticity here, with the only other mechanism for generation being the tangential stress boundary layer at the free surface (the flow simulated is slightly viscous). While having a physical origin, the vorticity magnitude near the free surface, especially before breaking (Fig. 22a), is likely to be overestimated: practical particle resolutions are insufficient to resolve vorticity generation within the tangential stress boundary layer, and free-surface kernel truncation also decreases the accuracy of gradient approximations in this region. Nevertheless, away from the free surface and in the presence of full kernel support (and increased accuracy), irrotationality is clearly well-maintained. From a loading point of view, the important characteristics of the jet are its shape, velocity, and pressure and comparison with experiment indicates that this is at least qualitatively reasonable while actually contributing little to overall force.

\subsubsection{Case F13: $H=0.1 \mathrm{~m}$}

Case F13 considers a steep wave of intermediate wave height $(H=0.1 \mathrm{~m})$ and high frequency $(f=1.22 \mathrm{~Hz})$. As can be seen in Fig. 23, steep sharp wave crests form, with some evidence of spilling at the crest. Reasonable agreement in the free-surface elevation is shown in Fig. 24 and subsequent force comparisons (Fig. 25) are in good agreement with 

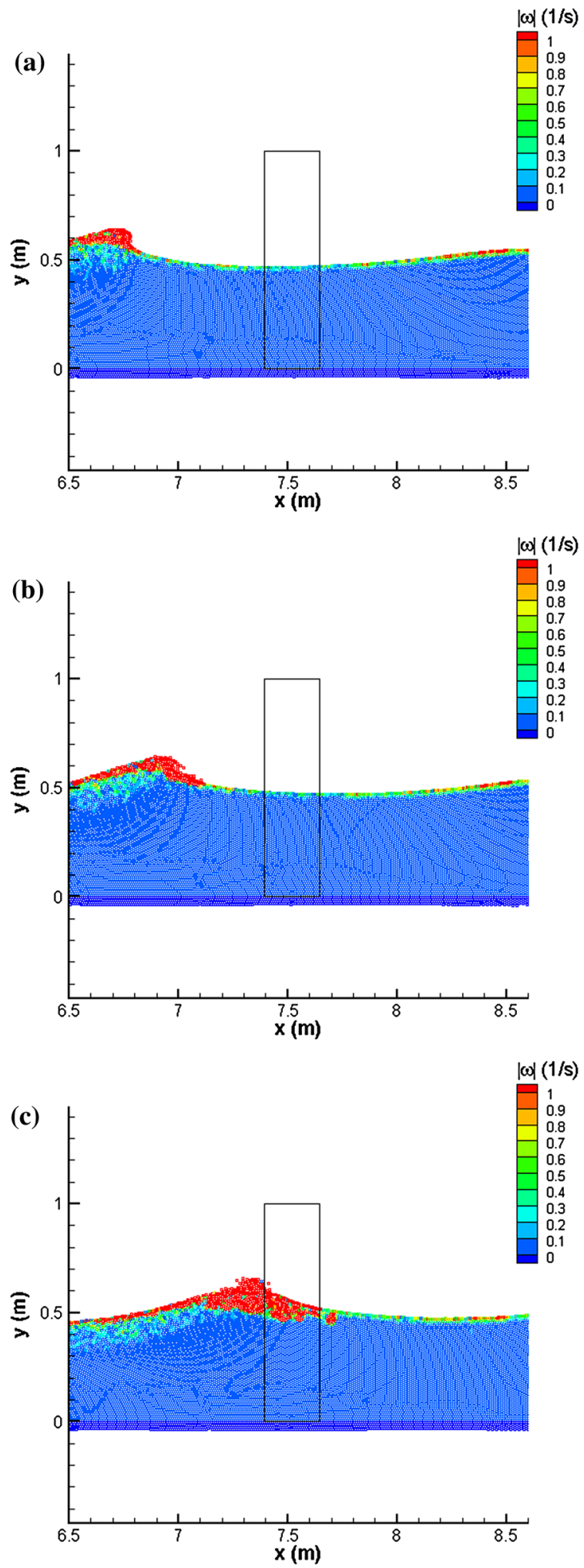

Fig. 22 As Fig. 20, but for vorticity magnitude. a $t=11.70 \mathrm{~s}, \mathbf{b} t=$ $11.83 \mathrm{~s}, \mathbf{c} t=11.96 \mathrm{~s}$

experiment for the first few crest interactions after the initial start-up waves. Given the ability of the SPH-FK approximation to provide reasonably accurate forces with roller impact

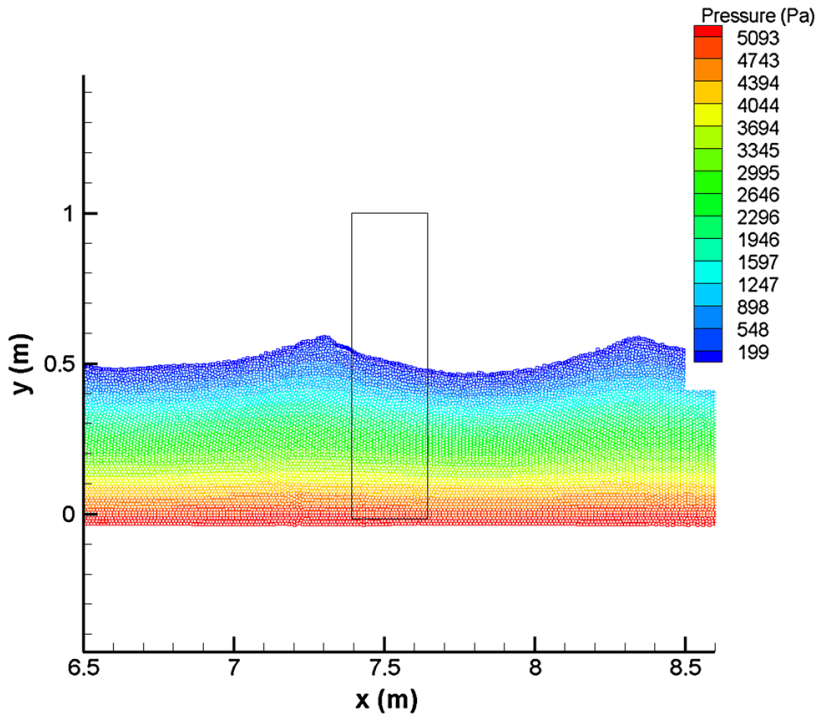

Fig. 23 Wave profile and pressure contours near the focal point for the F13 case $(t=20.93$ s $)$

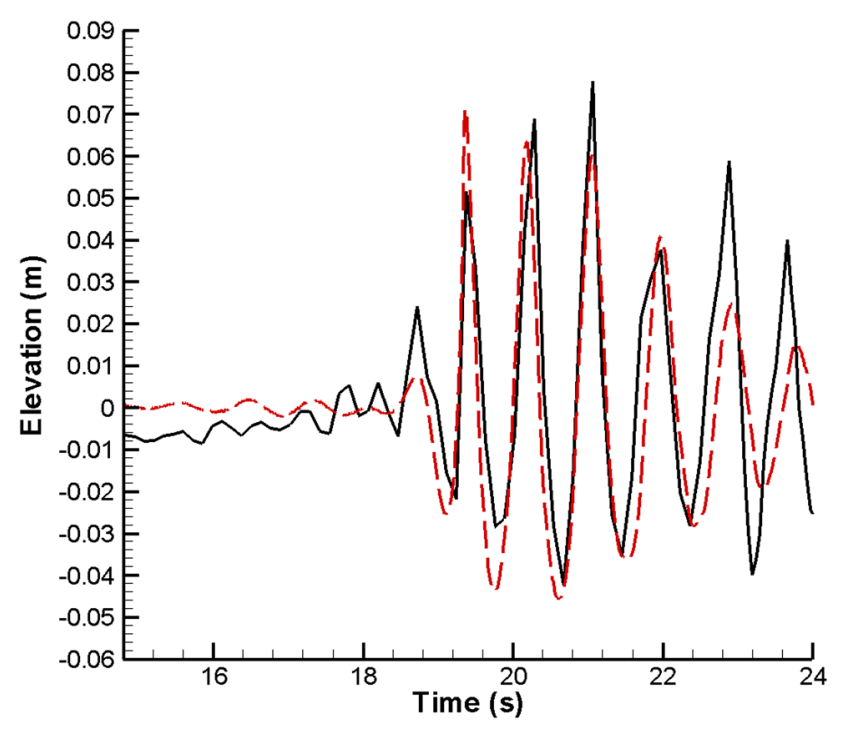

Fig. 24 Comparison between experimental and numerical results for the free-surface elevation at the front of the cylinder for focused wave case F13. The dashed lines denote experimental measurements, the black line is ISPH

(Fig. 19), the reasonable predictions provided in the case of spilling breakers is quite expected.

\subsubsection{Case F15: $H=0.22 \mathrm{~m}$}

The final focused wave case considered is that which results in a plunging breaker with jet impact direct on the cylinder (F15). Figure 26 shows the formation of the breaking wave (with pressure contours) at selected times during impact with the cylinder. The wavemaker input is calibrated and subsequently provides good agreement in the wave elevation at 


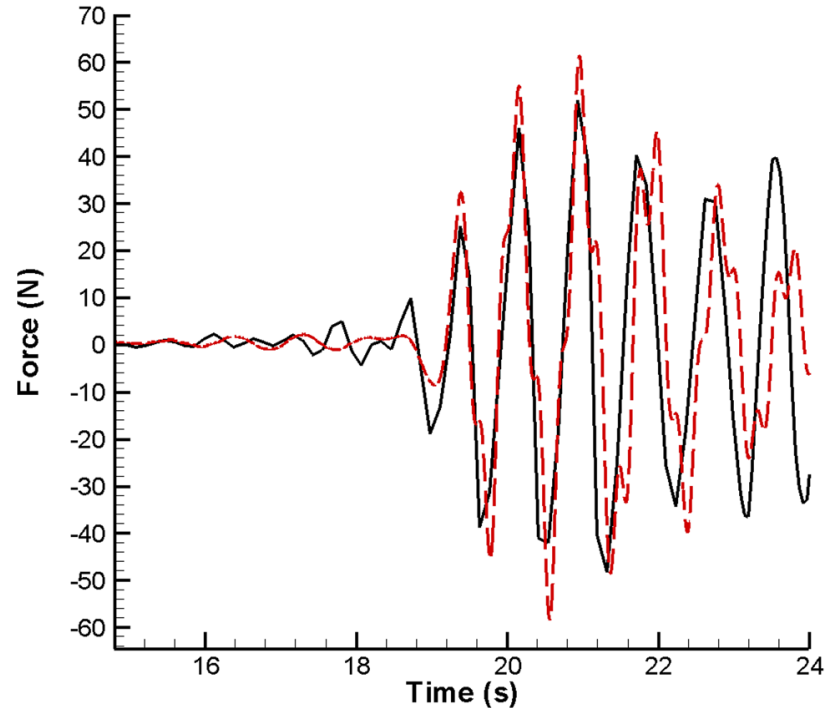

Fig. 25 Comparison between experimental and numerical results for the total horizontal force on the cylinder for focused wave case F13. The dashed lines denote experimental measurements, the black line is ISPH

the cylinder front (Fig. 27), especially before and up to the plunging jet impact at $t \approx 11.8 \mathrm{~s}$. Quite remarkably, force measurements up to and including the plunging jet impact are also well predicted by the FK modelling (Fig. 28). At later times agreement worsens, but this is to be expected as full cylinder interaction would be required for accurate modelling after such an impact. This is a case of considerable interest and hitherto has been argued to be the case potentially most damaging due to the high-speed impact of the plunging jet. For example, Zhou et al. (1991) consider plunging breaker impacts on cylinders and noted local impulsive impact pressures, although highly scattered, as high as $93 \mathrm{kPa}$ or $9 \mathrm{~m}$ of water. Despite the relatively high velocities of the plunging jet $[O(2 \mathrm{~m} / \mathrm{s})]$ (see Fig. 29), it seems that in this case a consideration of the undisturbed flow field alone is sufficient to get reasonable agreement in the total loading on the cylinder-including at jet impact at $t \approx 11.8 \mathrm{~s}$. Similarly to the discussion for case F14, the pressure in the jet will be near atmospheric and so will contribute little to the FK force relative to the sub-surface irrotational flow. Given the good agreement with experiment, any momentum imparted to the cylinder from the plunging jet must then be small compared to overall wave loading. Impulsive pressures exerted by the plunging jet may be significant locally, but these are very short-lived with rise times less than $1 \mathrm{~ms}$ (Zhou et al. 1991; Lind et al. 2015). Given the high degree of variability, locality, and short-lived nature of the impulsive impact pressures (which are also dependent on local air effects), it could be argued that their influence on structural integrity (in a global
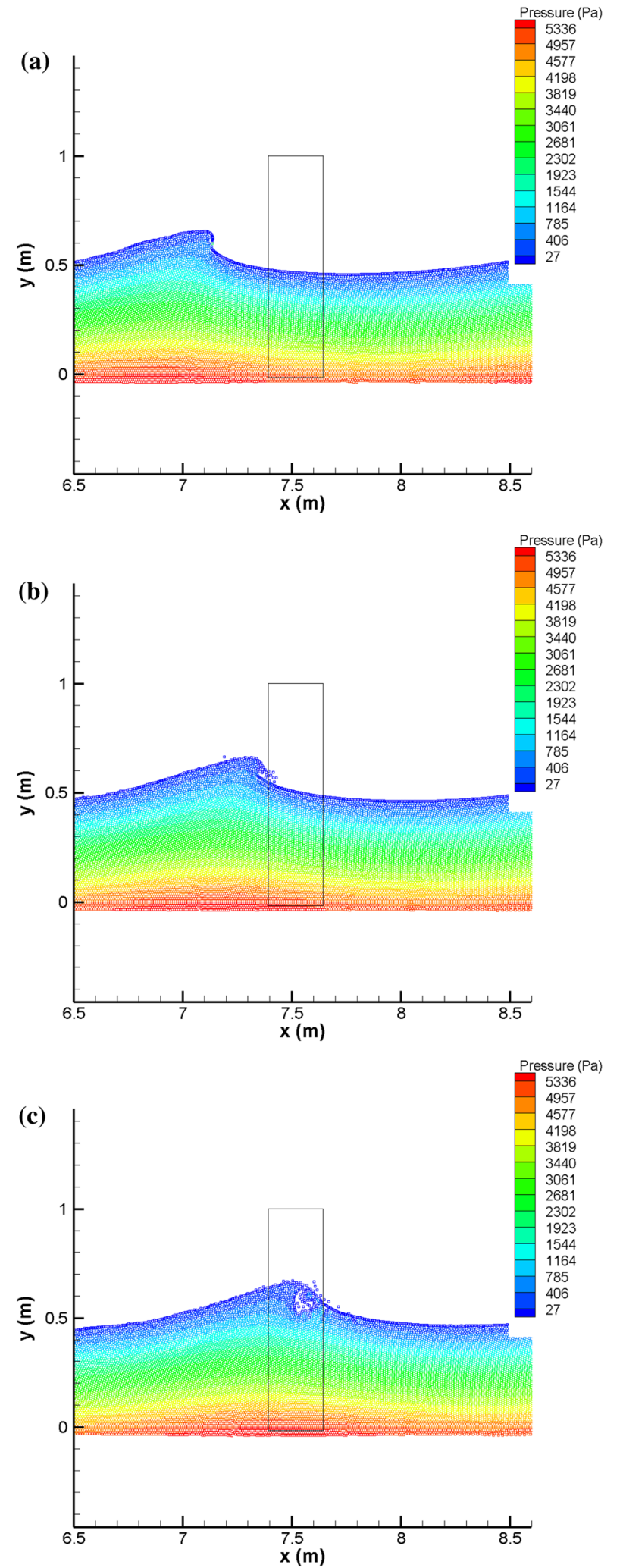

Fig. 26 Wave profile and pressure contours at select times near the focal point at the cylinder. a $t=11.70 \mathrm{~s}, \mathbf{b} t=11.83 \mathrm{~s}, \mathbf{c} t=11.96 \mathrm{~s}$ 


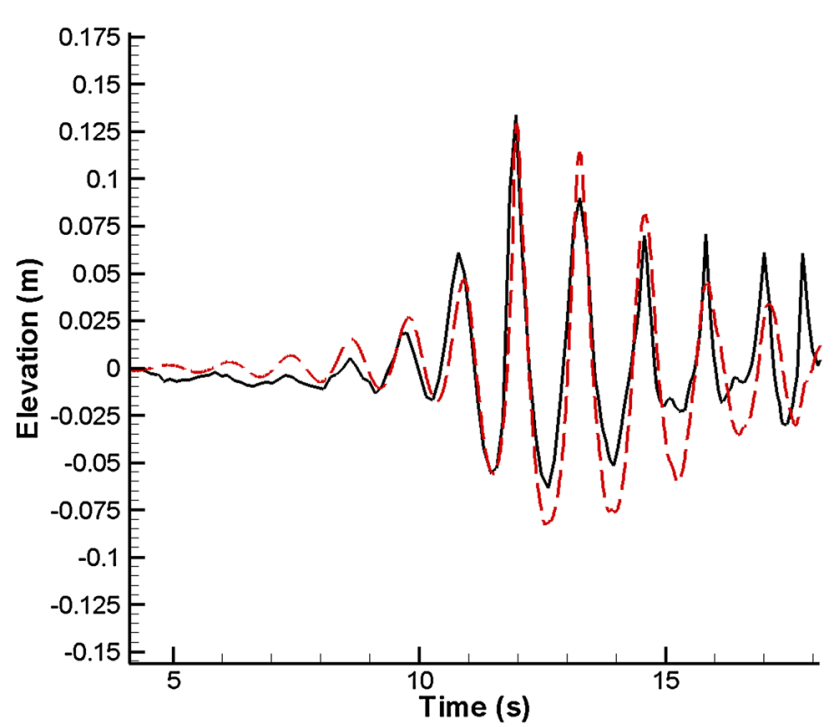

Fig. 27 Comparison between experimental and numerical results for the free-surface elevation at the front of the cylinder for focused wave case F15. The dashed lines denote experimental measurements, the black line is ISPH

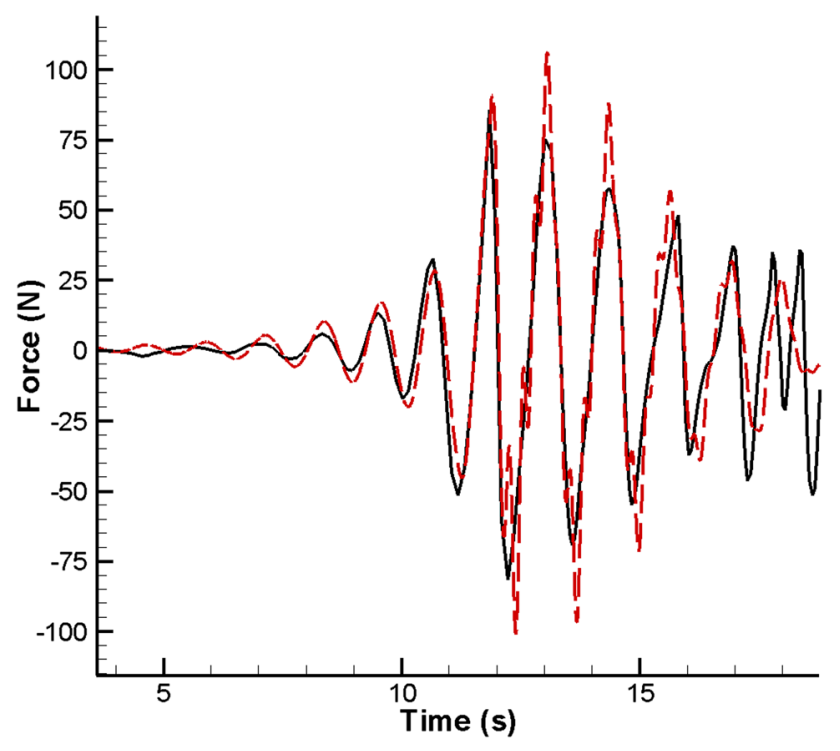

Fig. 28 Comparison between experimental and numerical results for the total horizontal force on the cylinder for focused wave case F15. The dashed lines denote experimental measurements, the black line is ISPH

sense) may be small compared to the more slowly varying (and persistent) total wave loads exerted. This is clearly an important topic that deserves greater attention and will be a subject for more detailed investigation.

The ability of the FK approximation to provide adequate force calculation, even for plunging breakers, is supported by Fig. 30 which shows force predictions for several spatial res-
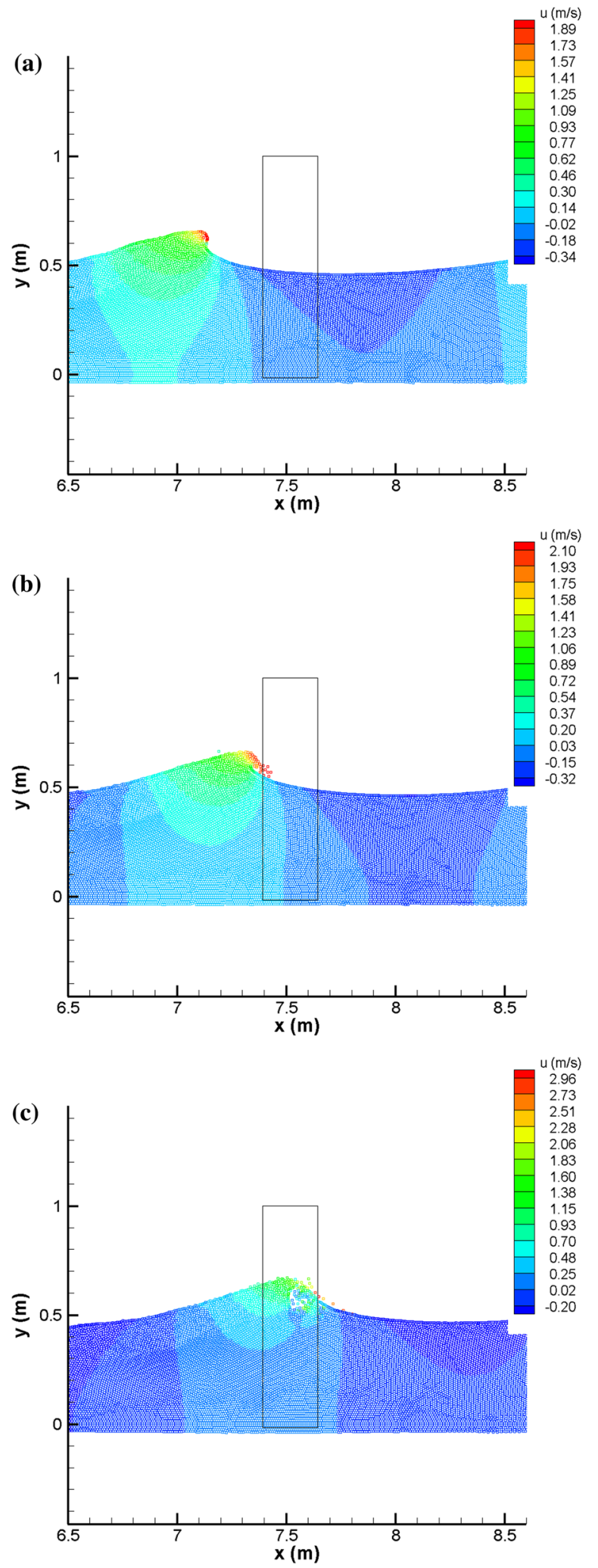

Fig. 29 As above (Fig. 26), but with contours displaying horizontal velocity. a $t=11.70 \mathrm{~s}, \mathbf{b} t=11.83 \mathrm{~s}, \mathbf{c} t=11.96 \mathrm{~s}$ 


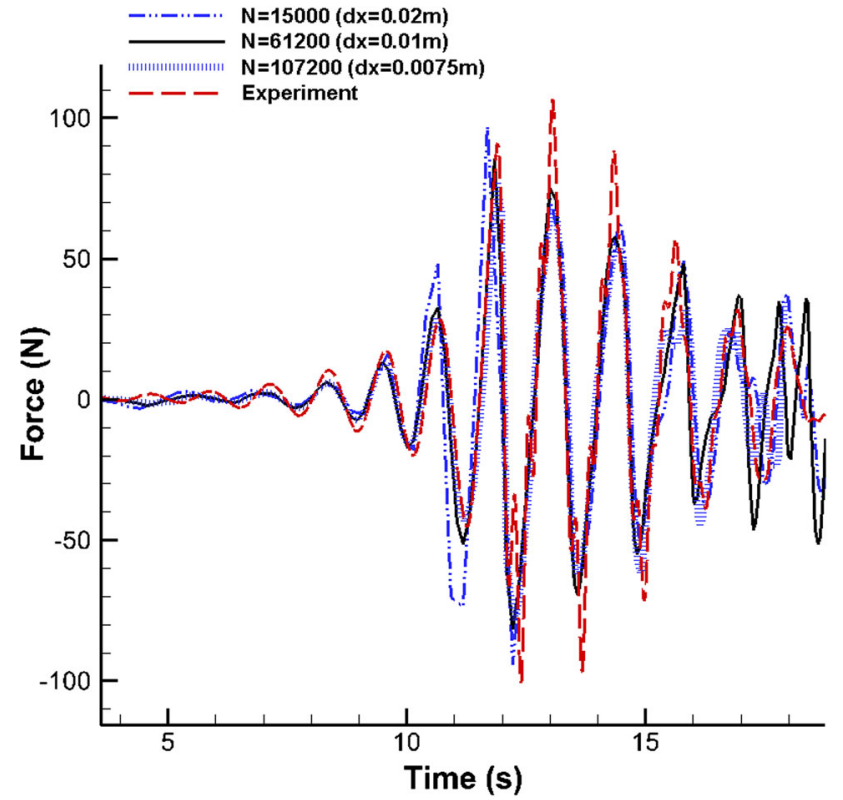

Fig. 30 ISPH force comparison with experimental focused wave data (case F15) for different particle resolutions

olutions, $d x$. All provide good agreement with experiment at the point of plunging breaker impact (even the coarsely refined spacing, $d x=0.02 \mathrm{~m}$ ) and there is little difference in results for refinement beyond $d x=0.01 \mathrm{~m}$, indicating that this resolution is sufficient even in this supposedly challenging test case.

It has been discussed in Sect. 3.2 and observed in recent experimental work (Stansby et al. 2013), that breaking wave impact exerts significant additional loading on the cylinder compared to an equivalent non-breaking case. This raises an interesting point regarding the stages of breaking where the maximum loading occurs. Early experimental works have recorded maximum forces due to the post-breaking roller (Miller et al. 1974). In contrast, recent experimental (Wienke and Oumeraci 2005) and numerical work (Hildebrandt and Schlurmann 2012) reports the maximum force on the cylinder at the point of overturning-where the plunging jet [or breaker tongue as in Wienke and Oumeraci (2005)] has a horizontal velocity and the front of the wave crest is vertical. This also aligns with the well-known empirical wave breaking study of Goda et al. (1966), which itself is based upon the classical works of Von Karman (1929) and Wagner (1932). A key benefit of the Froude-Krylov approximation is that the force on the cylinder can be calculated rapidly given a predetermined flow field. The flow is undisturbed by the cylinder so multiple cylinder locations and orientations can be considered rapidly from a single simulation.

Accordingly, for the case F15, the cylinder position is moved incrementally about the original experimental position $x_{c}=7.52 \mathrm{~m}$, to vary the form of the breaking wave at

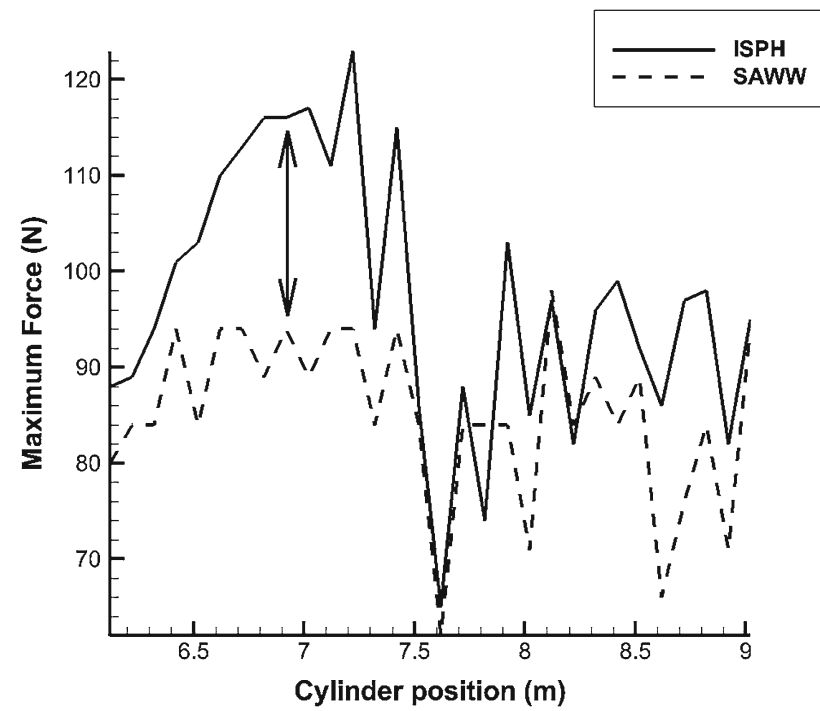

Fig. 31 Maximum predicted loads on the cylinder for various cylinder locations based on ISPH and SAWW. ISPH results are those from the breaking event of Fig. 26

impact. For each cylinder location, the local wave height at the cylinder is determined and input into the SAWW program to determine the loading at that wave height for the equivalent fully non-linear but symmetric (non-breaking) wave profile. Figure 31 plots the maximum horizontal force determined from ISPH and SAWW against various cylinder positions. There is clearly an amplification region $(6.5 \lesssim x \lesssim 7.5 \mathrm{~m})$, as highlighted approximately by the arrow, that produces an increased load for ISPH resulting purely from asymmetry in the impacting wave crest. From a symmetric to asymmetric (breaking) profile at fixed wave height, forces are amplified by as much as 1.3 approximately. This amplification factor is in quantitative agreement with the experimental assessment of Stansby et al. (2013), which reports amplification factors for various dimensionless depths, $k D$. This detail is presented in Fig. 32; the ISPH prediction is included and lies very close to the trendline through the experimental data (Stansby et al., 2013). From Fig. 31 the globally maximum force occurs at a cylinder location $x_{c}=7.22 \mathrm{~m}$, which corresponds to an impact as displayed in Fig. 33, where the wave is at the point of overturning and the wave front is vertical. This supports the empirical work of Goda et al. (1966), the numerical study of Hildebrandt and Schlurmann (2012), and the experimental findings of Wienke and Oumeraci (2005), where maximum forces were observed with impact at the point of overturning when newly formed jets remain horizontal. It is understandable that such a wave interaction may exert the largest load, as within a small time $d t$, such a profile imparts the largest possible horizontal momentum by displacing the largest possible mass of fluid, elements of which travel at velocities above the wave celerity (see the horizontal velocity contours of Fig. 33). This helps to explain the 


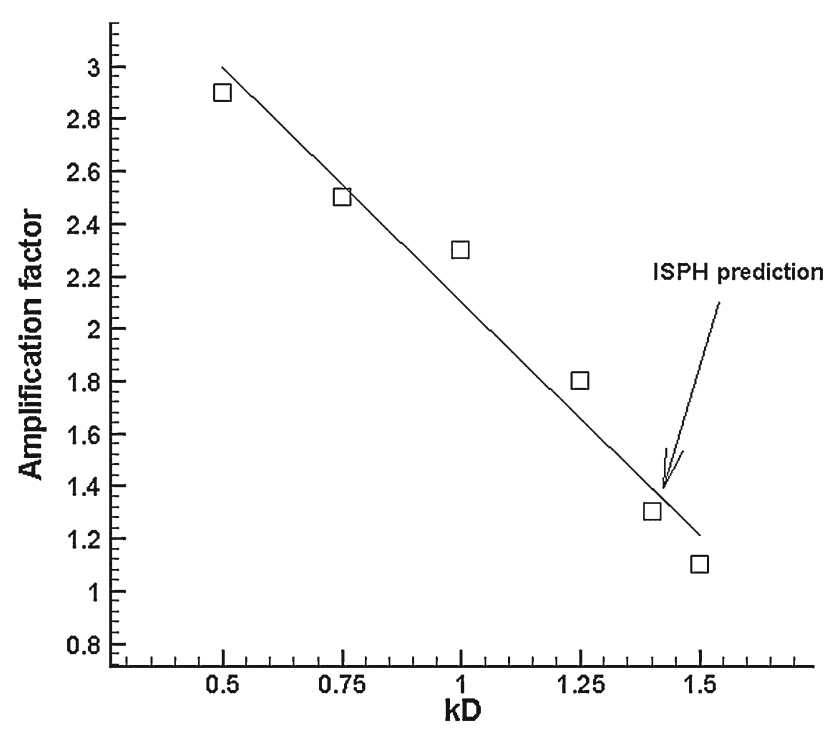

Fig. 32 Experimentally determined amplification factor values with $k D$ from Stansby et al. (2013). The black line is a linear trendline (least squares regression) and the numerical ISPH prediction is highlighted

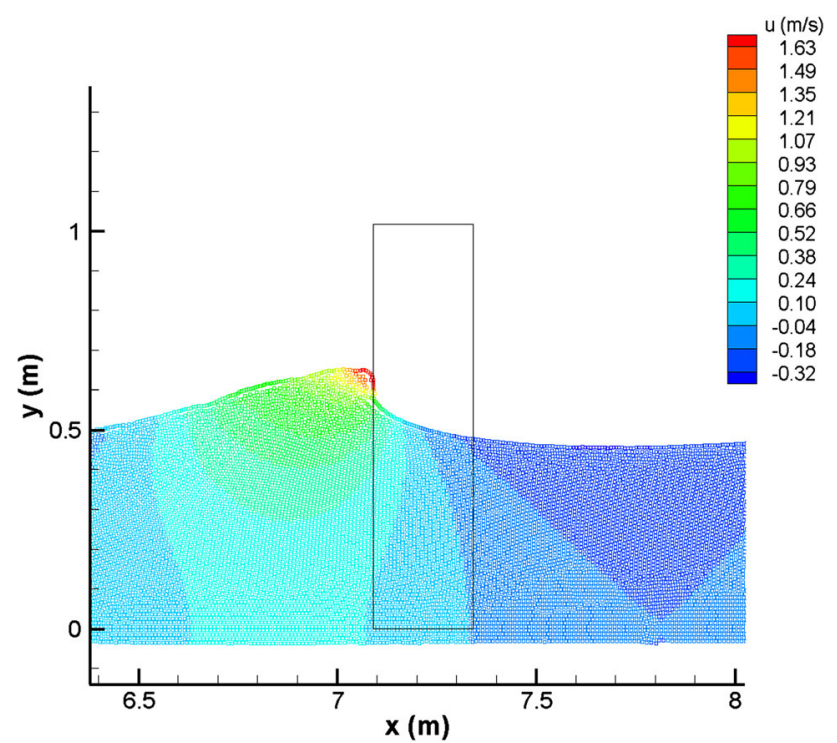

Fig. 33 The wave profile that exerts the largest load on the cylinder during the breaking process as determined through ISPH predictions. Contours denote the horizontal velocity values

increase observed over the equivalent symmetric non-linear impact provided by SAWW.

\section{Results: Part 2. Wave forces on a Taut Moored body}

Given the good approximations provided by the method for horizontal forces on a fixed vertical cylinder, the more challenging case of a moored floating body is considered. Results are compared with the experimental results of Hann
Table 3 Lengths and draft of the two mooring configurations studied

\begin{tabular}{lllll}
\hline Mooring Config. & $l_{s}(\mathrm{~m})$ & $l_{r}(\mathrm{~m})$ & $x_{m 0}(\mathrm{~m})$ & $D_{b}(\mathrm{~m})$ \\
\hline 1 & 0.63 & 1.38 & 0.27 & 0.31 \\
2 & 0.15 & 1.96 & 0.11 & 0.35 \\
\hline
\end{tabular}

Here $l_{s}$ is the unextended spring length, $l_{r}$ is the length of near inextensible Dyneema rope, and $x_{m 0}$ is the initial spring extension under hydrostatic forces. Initially then, the total mooring line length is $l_{m}=l_{s}+l_{r}+x_{m 0}$

et al. (2015) for a single-body moored wave energy converter (with some additional unpublished data provided by Hann directly). A schematic of the moored buoy setup is provided in Fig 2. Two mooring configurations are considered, both consisting of an elastic spring (unextended length, $l_{s}$; spring constant, $k=64 \mathrm{~N} / \mathrm{m}$ ) connected in series with a near inextensible rope (Dyneema rope) (unextended length, $l_{r}$; spring constant $k=35 \times 10^{3} \mathrm{~N} / \mathrm{m}$ ). The lengths and initial spring extensions for each configuration are presented in Table 3, alongside the buoy draft. In mooring configuration 1, the spring is long enough to allow a smooth extension of the mooring during wave interaction. In mooring configuration 2 , the spring is shortened and limited to a maximum length of $0.406 \mathrm{~m}$ by a parallel arrangement of four Dyneema ropes of equivalent spring constant $k_{\text {eq }}=28 \times 10^{3} \mathrm{~N} / \mathrm{m}$. In limiting the extension, the second configuration encourages "snatch" loads: large, short-lived mooring tensions reflective of the loads experienced in extreme wave conditions. The second (or snatch) mooring configuration is studied in both breaking and non-breaking waves.

With regard to the wave and basin properties, a constant still water depth of $D=2.8 \mathrm{~m}$ is used in a basin of length $L=30 \mathrm{~m}$. A piston wavemaker generates a focused wave group based on the Pierson-Moskowitz spectrum over a range $0.1-2 \mathrm{~Hz}$, with a peak frequency of $f_{p}=0.356 \mathrm{~Hz}$ and a peak amplitude of approximately $A_{N}=0.27 \mathrm{~m}$. These wave properties are used to generate the non-breaking wave group, with the breaking waves generated by multiplying the peak frequency by a factor 1.6 (as in experiment). The SPH discretisation uses a particle spacing of $d x=0.05 \mathrm{~m}$. The buoy geometry is defined as in Fig. 2, with $R_{b}=H_{b}=0.25 \mathrm{~m}$ and $d_{c}=0.5$. The buoy has a mass of $43.2 \mathrm{~kg}$ with a moment of inertia given by $1.61 \mathrm{kgm}^{2}$.

\subsection{Mooring configuration 1: non-snatch loads}

This mooring configuration has a longer elastic spring to enable smooth extension of the mooring line during wave interaction. This configuration just considers the nonbreaking wave case and Fig. 34 displays comparisons of experimental and numerical results for the heave motion of the buoy at the focal point (determined to be at $x_{f}=20 \mathrm{~m}$, 


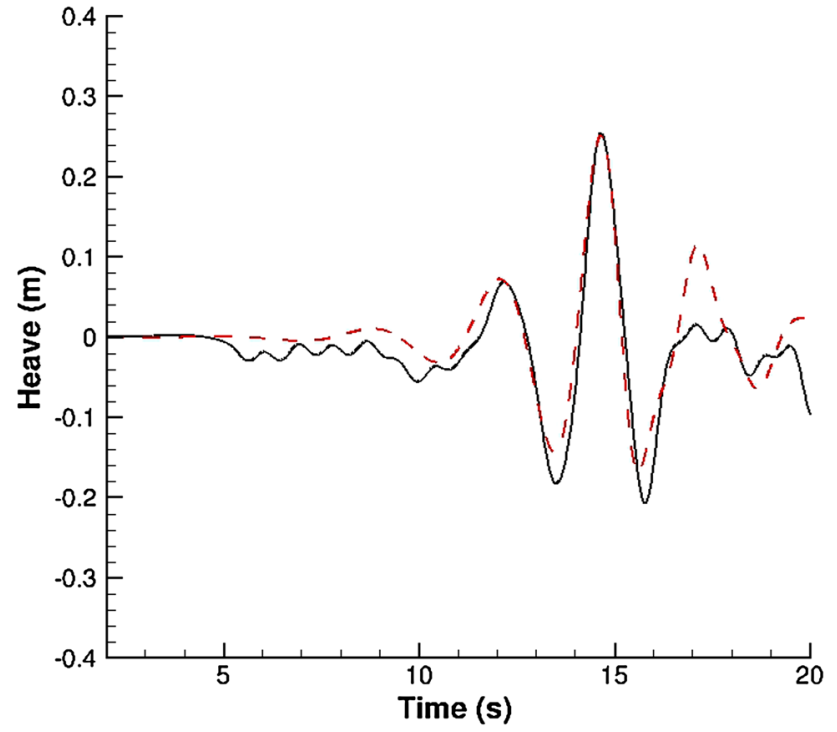

Fig. 34 Comparison of experimental and numerical predictions of the heave motion of the buoy during non-breaking focused wave interaction. Experimental results are denoted by the dashed line

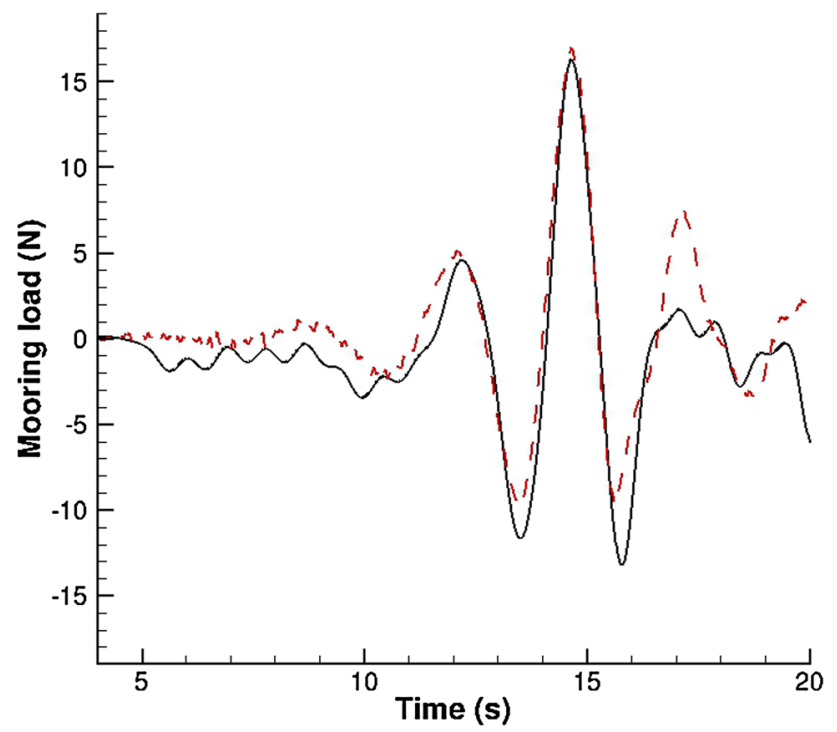

Fig. 35 Comparison of experimental and numerical predictions of the mooring load on the buoy during non-breaking focused wave interaction. Experimental results are denoted by the dashed line

approximately). Evidently, there is close agreement in the early stages of the interaction, including with the main crest. The agreement then deteriorates as the wave group passes through the focal point. This good agreement with the initial incident crests of the wave group is then reflected in the mooring load which displays near-identical behaviour qualitatively (see Fig. 35).

The low sensitivity to added mass is now demonstrated, with the mooring load recalculated using the complete force equation [Eq. (22) with $\mathbf{C}_{\mathbf{a}} \neq \mathbf{0}$ ] for the same incident wave

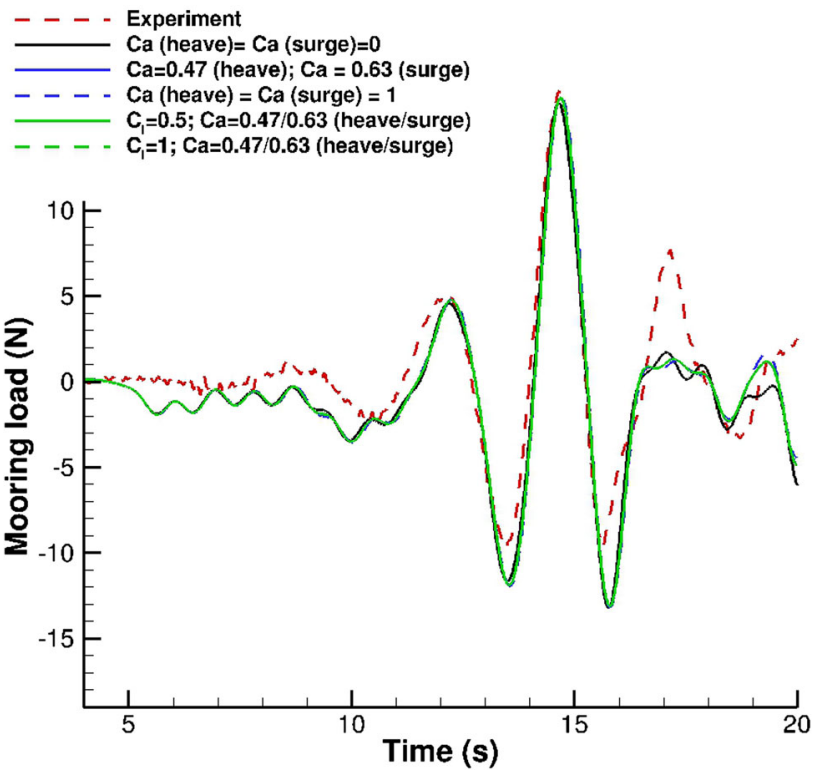

Fig. 36 Demonstration of insensitivity in the non-snatch mooring load to a selection of buoy added mass values

group for a selection of translational and rotational added mass value combinations (including the translational values determined by WAMIT). As can be seen in Fig. 36, there is little variation in the mooring load for a moderate (but realistic) range of added mass values. This supports the hypothesis that the effective force due to relative motion of the buoy to the surrounding fluid is suitably small compared to other hydrodynamic forces so as to enable added mass effects to be neglected, at least as a convenient first approximation.

\subsection{Mooring configuration 2: snatch loads}

This second buoy case considers a mooring configuration with spring/rope lengths combined as detailed in Table 3. The key difference here is that the easily extensible spring is limited in its extension (to a total length of $0.406 \mathrm{~m}$ ), allowing snatch loads to be exerted as the mooring reaches its maximum extension while interacting with the same focused wave group as mooring configuration 1 . This case now has two regimes of mooring response: the first is that governed by the elastic spring (with experimental spring constant $k=64 \mathrm{~N} / \mathrm{m}$ ) before the extension limit is reached; the second is the snatch regime when the elastic spring is at maximum extension, with any further extension necessarily appearing in the rope system comprising the rest of the mooring. The rope system that limits further spring extension consists of four Dyneema ropes in parallel, also connected to the main mooring rope in series. The experimentally determined value of the spring constant for a single rope is $35 \mathrm{~N} / \mathrm{mm}$, with the equivalent spring constant for the aforementioned system given by $k_{\mathrm{eq}}=28 \mathrm{~N} / \mathrm{mm}$. To obtain good experimental 


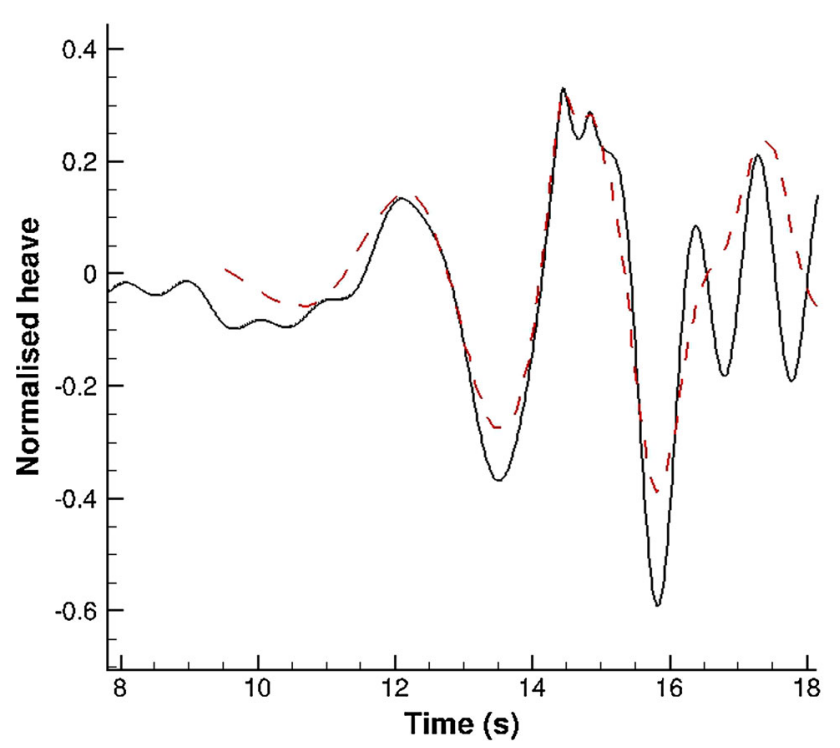

Fig. 37 Comparison of experimental and numerical predictions of the heave motion of the buoy during non-breaking focused wave interaction for a snatch enabled mooring configuration. Experimental results are denoted by the dashed line and displacement is normalised by the body diameter

agreement in the snatch regime, it was found necessary to include a spring damping term for the Dyneema rope interaction only (see Eq. 25). The damping ratio has not been defined by experiment but has been determined numerically to be approximately $\zeta=0.175$, indicating that, in reality, the rope component of mooring system deviates somewhat from a perfect theoretical spring. Note, the aforementioned value of $\zeta$ indicates a small amount of under-damping.

Figure 37 presents a comparison of numerical and experimental results for the heave motion of the buoy for the snatch load non-breaking wave case. Once again, the agreement is remarkably good over the first few wave crests, with the numerical method even approximating the small fluctuation in the heave at the main crest interaction (at $t \approx 14.6 \mathrm{~s}$ ). The corresponding mooring load is shown in Figure 38. Using experimentally determined spring constants and a damping ratio of $\zeta=0.175$ in the snatch regime, a good agreement is obtained with regard to position, magnitude, and duration of both snatch peaks. Note that, as in experiment, the loads are now normalised using the initial at rest mooring tension $(9.4 \mathrm{~N})$. A better agreement in magnitude is obtained for the first snatch load over the second. Indeed, it is the second snatch load that shows greater sensitivity to the damping ratio, as seen in Fig. 39. Without a damped response in the snatch mooring, the second load is predicted to be larger than the first.

While the non-breaking case demonstrates a good agreement with experiment for the snatch loads, the predictions worsen in the case of breaking waves. During plunging wave breaking, a series of snatch loads are observed, the number

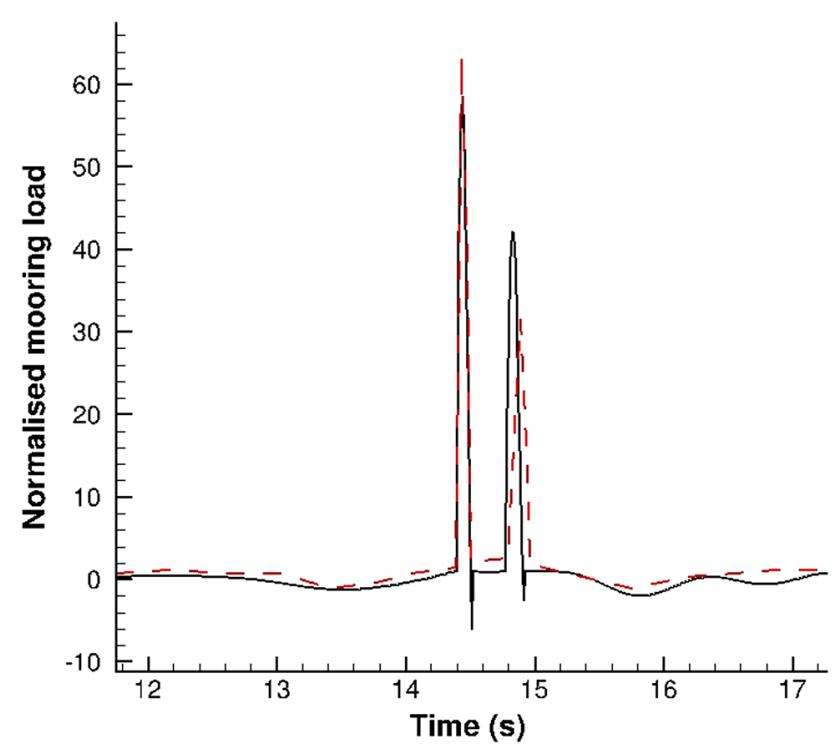

Fig. 38 Comparison of experimental and numerical predictions of the mooring load on the buoy during non-breaking focused wave interaction for a snatch enabled mooring configuration. Experimental results are denoted by the dashed line

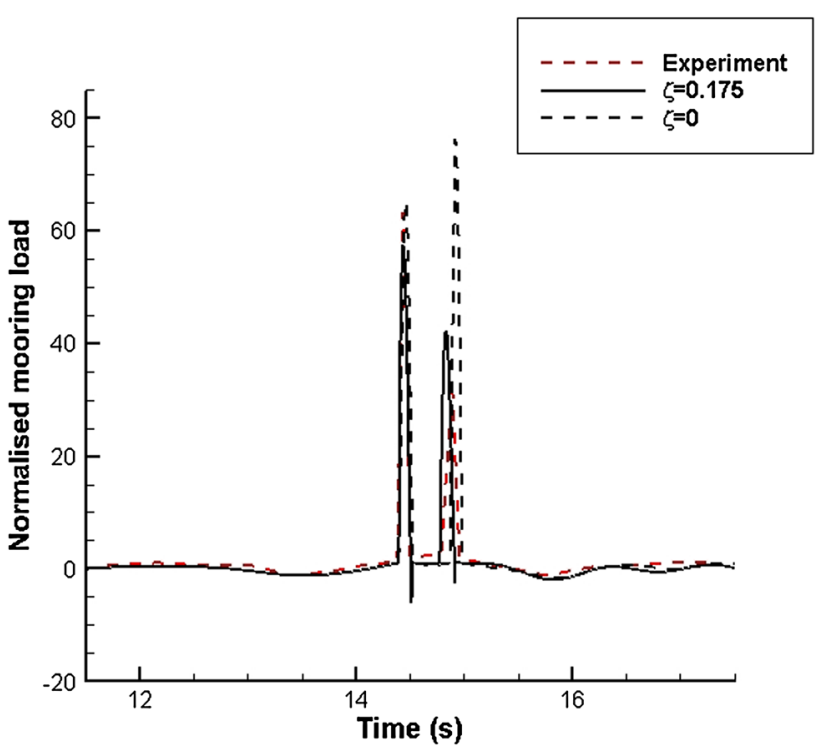

Fig. 39 Comparison of predicted snatch loads for different damping ratios, presented alongside experiment

and magnitude of which being dependent on the position of the device relative to the plunging breaker. With breaking sufficiently close to the buoy location, the first snatch load is due to interaction with the breaker, and this load decreases in magnitude to zero as the buoy moves upstream and distance from the plunging breaker increases. Figure 40 shows comparisons of the magnitude of the plunging breaker snatch load for a selection of buoy (initial) positions around the point of breaking (defined as the point of largest wave elevation and determined from the numerical simulations to be 


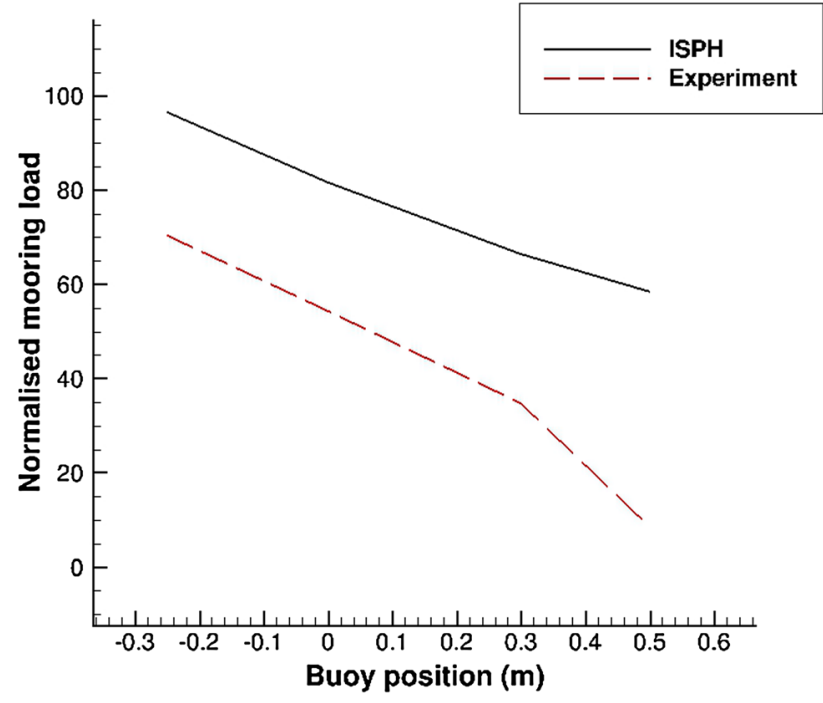

Fig. 40 Comparison of experimental and numerical measurements for the magnitude of the first snatch mooring load due to a plunging breaker. The load is plotted against the initial position of the centroid of the buoy relative to the point of breaking at $x \approx 13.5$

at $x=13.5 \mathrm{~m}$, approximately). As distance from the point of breaking increases, the load decreases at mostly the same rate as experiment, but the magnitudes are over-predicted by around $30 \%$ in most cases. Any further snatch loads exerted in post-breaking are not very well predicted in either location or magnitude.

Indeed, the flow field is likely to be sufficiently complex in late post-breaking that good agreement should not be expected from a numerical method that is close to incompressible, inviscid, and based on an undisturbed flow field. In reality, the flow will be significantly aerated and altered by buoy motion, with significant energy loss following breaking. Indeed, all results in this study affirm that the best predictions are observed only for the first few wave crest interactions, but, crucially, this includes the main crest where the most extreme loads occur. Further improvement may be possible with careful selection of added mass coefficients, including negative added mass values [observed in oscillating bodies at free surfaces (Mciver and Evans 1984)], which may also be useful in modelling aeration.

\section{Conclusions}

This paper presents an alternative and efficient numerical approach for the calculation of three-dimensional loads on fixed and floating bodies due to breaking and non-breaking waves. Fully non-linear wave profiles and dynamics are determined accurately using a state-of-the-art incompressible SPH method. Forces on the bodies are then determined from the undisturbed flow field using the Froude-Krylov force with added mass determined from empirical or the- oretical coefficients. For the cylinder case, two wave types are studied (regular and focused) and detailed comparisons are made with experimental data (Luck and Benoit 2004; Zang et al. 2010).

The Froude-Krylov approximation with theoretical added mass is quite accurate and is able to predict initial loads on cylinders due to waves in various stages of breaking. Results corroborate recently reported experimental (Wienke and Oumeraci 2005) and numerical (Hildebrandt and Schlurmann 2012) results that attribute the maximum load during breaking to when the wave is at the point of overturning, the plunging jet is still horizontal, and the wave front vertical. The results do indicate that wave asymmetry is most significant in amplifying force over that for steep symmetric waves rather than impact due to breaking jets.

Predictions for the buoy response and mooring load are in good agreement with experiment (Hann et al. 2015) for the non-breaking cases; both the snatch and non-snatch loads are well predicted using experimentally provided mooring line spring constants. With wave breaking, mooring load predictions are less accurate, but the snatch loads due to the first plunging breaker demonstrate the same dependence on relative buoy position as experiment, with a quantitatively similar rate of decrease with distance. Overall, the method has a number of benefits: the simulations are two-dimensional and of resolution only required for wave simulation, so computations are comparatively fast. Furthermore, forces on a body in multiple configurations can be undertaken rapidly as force calculations are done in post-processing on the undisturbed flow. With further validation, this approach may prove to be a useful computational tool for coastal/offshore engineering practice.

Acknowledgments The authors wish to thank Dr. Jun Zang (Bath), Dr. Martyn Hann and Prof Deborah Greaves (Plymouth) for the provision of focused wave experimental data for the cylinder and buoy (Bath and Plymouth, respectively). The authors would also like to thank Dr. Hanbin Guo for WAMIT calculations. Support through EPSRC Supergen Marine Grand Challenge Project X-MED EP/J010235/1 is also acknowledged.

Open Access This article is distributed under the terms of the Creative Commons Attribution 4.0 International License (http://creativecomm ons.org/licenses/by/4.0/), which permits unrestricted use, distribution, and reproduction in any medium, provided you give appropriate credit to the original author(s) and the source, provide a link to the Creative Commons license, and indicate if changes were made.

\section{References}

Aller AB (2015) Smoothed particle hydrodynamics model for civil and coastal engineering applications. $\mathrm{PhD}$ thesis, Universida de Vigo

Bonet J, Lok TSL (1998) Variational and momentum preservation aspects of smooth particle hydrodynamics formulations. Comput Methods Appl Mech Eng 43:785-819 
Bredmose H, Jacobsen NG (2010) Breaking wave impacts on offshore wind turbine foundations: focused wave groups and CFD. In: Proceedings of the 29th International Conference on Ocean Offshore Arctic Engineering, ASME, Shanghai, China

Bredmose H, Jacobsen NG (2011) Vertical wave impacts on offshore wind turbine inspection platforms. In: Proceedings of the 30th International Conference on Ocean Offshore Arctic Engineering, ASME, Rotterdam, The Netherlands

Buss GY, Stansby PK (1982) SAWW-a computer program to calculate the properties of steady water waves. Tech. rep., Simon Engineering Laboratories, University of Manchester, Manchester, UK

Chan ES, Cheong HF, Tan BC (1995) Laboratory study of plunging wave impacts on vertical cylinders. Coast Eng 25:87-107

Chen L, Zang J, Hillis A, Morgan G, Plummer A (2014) Numerical investigation of wave-structure interaction using OpenFOAM. Ocean Eng 88:91-109

Chorin AJ (1968) Numerical solution of the Navier Stokes equations. J Math Comp 22:745-762

Christensen ED, Bredmose H, Hansen EA (2005) Extreme wave forces and run-up on offshore wind turbine foundations. In: Proceedings of the Copenhagen Offshore Wind Conference, Copenhagen, Denmark

Crespo AJC, Dominguez JM, Rogers BD, Gmez-Gesteira M, Longshaw S, Canelas R, Vacondio R, Barreiro A, Garca-Feal O (2015) DualSPHysics: open-source parallel CFD solver based on smoothed particle hydrodynamics (SPH). Comput Phys Commun 187:204216

Cummins SJ, Rudman M (1999) An SPH projection method. J Comput Phys 152:584-607

Dalrymple RA, Rogers BD (2006) Numerical modeling of water waves with the SPH method. Coast Eng 53(2):141-147

Dean RG, Dalrymple RA (1991) Water wave mechanics for engineers and scientists. In: Advanced series on ocean engineering, vol 2. World Scientific, Singapore

Farahani RJ, Dalrymple RA (2014) Three-dimensional reversed horseshoe vortex structures under broken solitary waves. Coast Eng 91:261-279

Goda Y (1970) A synthesis of breaker indices. Trans Japan Society of Civil Eng 2:227-230

Goda Y, Haranaka S, Kitahata M (1966) Study of impulsive breaking wave forces on piles. Rep Port Harbor Res Inst 5:1-30

Gotoh H, Khayyer A, Ikari H, Arikawa T, Shimosako K (2014) On enhancement of incompressible SPH method for simulation of violent sloshing flows. Appl Ocean Res 46:104-115

Grilli S, Guyenne P, Dias F (2001) A fully non-linear model for threedimensional overturning waves over an arbitrary bottom. Int $\mathrm{J}$ Numer Methods Fluids 35(7):829-867

Gui Q, Shao S, Dong P (2014) Wave impact simulations by an improved isph model. J Waterw Port Coast Ocean Eng 140(3):04014,005

Guo X, Lind SJ, Rogers BD, Stansby PK, Ashworth M (2013) Efficient massive parallelisation for incompressible smoothed particle hydrodynamics with $10^{8}$ particles. Proceedings of the 8th International SPHERIC Workshop, Trondheim, Norway, pp 397-402

Hann M, Greaves D, Raby A (2015) Snatch loading of a single taut moored floating wave energy converter due to focussed wave groups. Ocean Eng 96:258-271

Hildebrandt A, Schlurmann T (2012) Breaking wave kinematics, local pressures, and forces on a tripod support structure. Proceedings of the 33rd International Conference on Coastal Engineering, ICCE, Santander, Spain

Keulegan GH, Carpenter LH (1958) Forces on cylinders and plates in an oscillating fluid. J Res Nat Bur Stand 60:423-440

Khayyer A, Gotoh H (2011) Enhancement of stability and accuracy of the moving particle semi-implicit method. J Comput Phys 230(8):3093-3118
Khayyer A, Gotoh H (2013) Enhancement of performance and stability of MPS mesh-free particle method for multiphase flows characterized by high density ratios. J Comput Phys 242:211-233

Lee CH(1995) WAMIT theory manual. Massachusetts Institute of Technology, Cambridge, Massachusetts, USA

Lee ES, Moulinec C, Xu R, Violeau D, Laurence D, Stansby P (2008) Comparisons of weakly compressible and truly incompressible algorithms for the SPH mesh free particle method. J Comput Phys 227:8417-8436

Lind SJ, Xu R, Stansby PK, Rogers BD (2012) Incompressible smoothed particle hydrodynamics for free-surface flows: a generalised diffusion-based algorithm for stability and validations for impulsive flows and propagating waves. J Comput Phys 231(4):1499-1523. doi:10.1016/j.jcp.2011.10.027

Lind SJ, Stansby PK, Rogers BD, Lloyd PM (2015) Numerical predictions of water-air wave slam using incompressible-compressible smoothed particle hydrodynamics. Appl Ocean Res 49:57-71

Longuet-Higgins MS, Cokelet ED (1976) The deformation of steep surface waves on water. I. A numerical method of computation. Proc R Soc Lond A 350:1-26

Luck M, Benoit M (2004) Wave loading on monopile foundation for offshore wind turbines in shallow-water areas. In: Coastal Engineering 2004 World Scientific Publishing Co Pte Ltd, Lisbon, Portugal, p 4595

Ma QW, Yan S (2009) QALE-FEM for numerical modelling of nonlinear interaction between 3D moored floating bodies and steep waves. Int J Numer Methods Eng 78(6):713-756

Mciver P, Evans DV (1984) The occurrence of negative added mass in free-surface problems involving submerged oscillating bodies. J Eng Math 18(1):7-22

Miller RL, Leverette S, O’Sullivan J, Tochko J, Theriault K (1974) Field measurements of impact pressures in surf. Proceedings of the 14th Coastal Engineering Conference, p 1761

Monaghan JJ (1994) Simulating free surface flows with SPH. J Comput Phys 110(2):399-406

Morison JR, O'Brien MP, Johnson JW, Schaaf SA (1950) The force exerted by surface waves on piles. J Petrol Technol 2:149-154

Morison JR, Johnson J, O'Brien M (1953) Experimental studies of forces on piles. Proceedings of the 4th Coastal Engineering Conference, Chicago, USA, pp 340-370

Morris JP, Fox PJ, Zhu Y (1997) Modelling low Reynolds number incompressible flows using SPH. J Comput Phys 136:214-226

Nadaoka K, Hino M, Koyano Y (1989) Structure of the turbulent flow field under breaking waves in the surf zone. J Fluid Mech 204:359_ 387

Newman J (1977) Marine hydrodynamics. MIT Press, Cambridge, Massachusetts, USA

Oger G, Doring M, Alessandrini B, Ferrant P (2007) An improved SPH method: towards higher order convergence. J Comput Phys 225:1472-1492

Rienecker MM, Fenton JD (1981) A Fourier approximation method for steady water waves. J Fluid Mech 104:119-137

Sarpkaya T, Isaacson M (1981) Mechanics of wave forces on offshore structures. Van Nostrand Reinhold Co, New York

Schwaiger HF (2008) An implicit corrected SPH formulation for thermal diffusion with linear free surface boundary conditions. Int $\mathrm{J}$ Numer Meth Eng 75:647-671

Shao S (2006) Incompressible SPH simulation of wave breaking and overtopping with turbulence modelling. Int J Numer Methods Fluids 50(5):597-621

Stansby PK, Devaney LC, Stallard TJ (2013) Breaking wave loads on monopiles for offshore wind turbines and estimation of extreme overturning moment. Renew Power Gener IET 7(5):514-520

Taylor PH, Jonathan PP, Harland LA (1997) Time domain simulation of jack-up dynamics with the extremes of a Gaussian process. ASME J Vib Acoust 119(4):624-628 
Tromans PS, Anaturk AR, Hagemeijer P (1991) A new model for the kinematics of large ocean waves-application as a design wave. In: The First International Offshore and Polar Engineering Conference, International Society of Offshore and Polar Engineers, Edinburgh, UK

Von Karman T (1929) The impact of seaplane floats during landing. NACA Technical Note 321, Washington

Wagner H (1932) Über stoss-und gleitvorgänge an der oberfläche von flüssigkeiten. Z F Angew Math Mech 12(4):193-235

Watanabe A, Horikawa K (1974) Breaking wave forces on large diameter cell. Proceedings of the 14th International Conference on Coastal Engineering, Copenhagen, Denmark, pp 1741-1760

Westphalen J, Greaves DM, Raby A, Hu ZZ, Causon DM, Mingham CG, Omidvar P, Stansby PK, Rogers BD (2014) Investigation of wave-structure interaction using state of the art CFD techniques. Open J Fluid Dyn 4:18-43

Wiegel R (1982) Forces induced by breakers on piles. Proceedings of the 18th International Conference on Coastal Engineering, ASCE, Cape Town, South Africa, pp 1699-1715
Wienke J, Oumeraci H (2005) Breaking wave impact force on a vertical and inclined slender pile-theoretical and large-scale model investigations. Coast Eng 52:435-462

Xu R (2010) An improved incompressible smoothed particle hydrodynamics method and its application in free-surface simulations. $\mathrm{PhD}$ thesis, University of Manchester

Xu R, Stansby PK, Laurence D (2009) Accuracy and stability in incompressible SPH (ISPH) based on the projection method and a new approach. J Comput Phys 228:6703-6725

Zang J, Taylor PH, Morgan G, Stringer R, Orszaghova J, Grice J, Tello M (2010) Steep wave and breaking wave impact on offshore wind turbine foundations-ringing revisited. 25th International Workshop on Water Waves and Floating Bodies, Harbin

Zhou D, Chan ES, Melville WK (1991) Wave impact pressures on vertical cylinders. Appl Ocean Res 13(5):220-234 